\title{
Load Optimization With User Association in Cooperative and Load-Coupled LTE Networks
}

Lei You and Di Yuan

The self-archived version of this journal article is available at Linköping University Electronic Press:

http:// urn.kb.se/ resolve?urn=urn:nbn:se:liu:diva- 138272

N.B.: When citing this work, cite the original publication.

You, L., Yuan, Di, (2017), Load Optimization With User Association in Cooperative and Load-Coupled LTE Networks, IEEE Transactions on Wireless Communications, 16(5), 3218-3231. https:// dx.doi.org/ 10.1109/TWC.2017.2676762

Original publication available at:

https:// dx.doi.org/ 10.1109/TWC.2017.2676762

Copyright: Institute of Electrical and Electronics Engineers (IEEE)

http:// www.ieee.org/ index.html

(C) 2016 IEEE. Personal use of this material is permitted. However, permission to reprint/ republish this material for advertising or promotional purposes or for creating new collective works for resale or redistribution to servers or lists, or to reuse any copyrighted component of this work in other works must be obtained from the IEEE. 


\title{
Load Optimization with User Association in Cooperative and Load-Coupled LTE Networks
}

\author{
Lei You ${ }^{1}$ and Di Yuan ${ }^{1}$ \\ ${ }^{1}$ Department of Science and Technology, Linköping University, Sweden \\ \{lei.you; di.yuan@liu.se\}
}

\begin{abstract}
We extend the problem of optimizing user association for load balancing in cellular networks along two dimensions. First, we consider joint transmission (JT), which is one of the coordinated multipoint (CoMP) techniques, with which a user may be simultaneously served by multiple base stations. Second, we account for, mathematically, the coupling relation between the base stations' load levels that are dependent on each other due to inter-cell interference. We formulate two optimization problems, sum load minimization (MinSumL) and maximum load minimization (MinMaxL). We prove that both MinSumL and MinMaxL are $\mathcal{N} \mathcal{P}$-hard. We propose a mixed integer linear programming (MILP) based scheme by means of linearization. This approach also leads to a bounding scheme for performance benchmarking. Then, we derive a set of partial optimality conditions. Fulfillment of the conditions will guarantee performance improvement for both MinSumL and MinMaxL. A solution algorithm is then derived based on the conditions. Simulation results are provided to demonstrate the effectiveness of the approaches.
\end{abstract}

Keywords: joint transmission, load balancing, load-coupling, heterogeneous networks, resource efficiency.

\section{INTRODUCTION}

\section{A. Background}

To provide high rate and capacity in the fifth generation $(5 \mathrm{G})$ communication systems, efficient utilization of time-frequency resource is crucial. The efficiency of time-frequency resource units (RUs) is influenced by many aspects, including radio access, radio resource management, network planning, interference coordination/mitigation, and user association strategies. Inappropriate user association may result in inefficient usage of RUs. In particular, the user association policy is crucial in terms of balancing the resource usage among cells and avoid overloading, especially in heterogeneous networks (HetNets) scenarios due to the large difference between the transmit power levels of macro cells (MCs) and small cells (SCs).

In this paper, we extend the optimization problem of user association along two dimensions. The first is the consideration of coordinated multipoint (CoMP) transmission [2]. As one of the CoMP techniques, Joint Transmission (JT) allows a user equipment (UE) to simultaneously receive its data from multiple base stations (BSs) on the same set of time-frequency resource. Second, we account for mathematically the loadcoupling relation among the cells. Here, the load refers to the amount of utilized RUs, and this concept has been very useful for performance characterization [3]-[12]. The load in one cell governs the amount of transmission and consequently inter-cell interference. Thus, even for given association of the
UEs, the cell load levels cannot be treated independently from each other. Rather, they are inherently coupled, and constitute the so called load-coupling system that mathematically formulates the mutual influence. To our knowledge, this type of characterization has not been developed for JT.

\section{B. Related work}

User association optimization subject to quality of service (QoS) constraints has been studied in [13]-[18] for non-JT scenarios. In [13], the authors applied Lagrangian duality for min-max fairness. Under the same performance objective, the authors of [14] studied BS association and power allocation. In [15], optimal user association for ultra-dense networks has been approached by means of integer linear programming. User association with the perspective of random HetNets, where access points are distributed according to a stationary point process, has been studied in [16]. It is shown that, if the association is based on received power, the amount of association to SCs decreases with the path loss exponent and increases with the channel gain variance. In [17], intracell cooperation using relays for resource allocation optimization is investigated. By using the KKT conditions, the problem is decomposed into several, independent sub-problems. User association and resource allocation with the presence of possible misreporting of information from individual users have been addressed by game theory in [18].

Radio resource allocation for CoMP has been studied in [19]-[21]. In [19], the authors considered optimizing user association for load balancing, in which the load refers to the number of users associated to the cell, and thus load balancing implicitly assumes that all users are uniform in resource consumption. The study in [20] applied stochastic geometry for analyzing network coverage with cooperative transmission. Resource allocation in non-coherent JT-CoMP scenarios has been studied in [21], by optimizing a fractional frequency reuse scheme.

\section{Our Work}

There is a lack of investigation that accounts for the loadcoupling relation with $\mathrm{JT}$ in the context of user association. The load-coupling model, in which the load represents the amount of time-frequency resource consumption, rather than counting the number of users, enables an analytical characterization of the user-specific resource requirement, and the 
dynamic dependence relation of resource consumption among cells due to mutual interference [3], [8], [9], [11].

The load-coupling model consists in a group of non-linear equations. Accounting for JT adds to the complexity of loadcoupling analysis. We study optimizing the cell-UE association with JT and cell-load-coupling. We consider two performance metrics. The first is the total amount of time-frequency resource consumption, i.e., sum of cell load levels. The second metric is the minimization of maximum load, for the effect of load balancing among cells.

Our main contributions are as follows. We extend and generalize the load-coupling model proposed in [4] to JT, and formulate both the sum load minimization (MinSumL) and the maximum load minimization (MinMaxL) with the new model. Both MinSumL and MinMaxL are proved to be $\mathcal{N} \mathcal{P}$ hard. We first provide a solution approach based on a linearization of the non-linear load coupling constraint. The two problems are then approximately represented by two MILPs. The approach also yields a bounding scheme for performance benchmarking. In our second approach, we derive a set of partial optimality conditions; fulfillment of the conditions will guarantee performance improvement for both MinSumL and MinMaxL. These conditions provide a theoretical basis for deriving a solution algorithm referred to as Minimization of Load (MinL), for optimizing the association between cells and UEs for MinSumL and MinMaxL. We present extensive results to demonstrate the effectiveness of the solution approaches as well as the benefit of JT in load optimization.

\section{System Model AND LOAd COUPLing}

\section{A. Notations}

We now generalize the model in [3]-[11] to JT. Denote the set of cells by $\mathcal{J}$ and the set of UEs by $\mathcal{J}$. Let $n=|\mathcal{J}|$ and $m=|\mathcal{J}|$. Denote by $\mathcal{J}_{j}^{+}$the set of candidate serving cells of UE $j$. For any UE $j$, denote by $\mathcal{J}_{i}^{+}$the set of UEs that potentially can be served by cell $i$. Apparently, $i \in \mathcal{J}_{j}^{+} \Leftrightarrow j \in \mathcal{J}_{i}^{+}$. Each UE $j$ is assigned with a home cell $c_{j}$, which is chosen as the cell with the best received power at UE $j$. The union of all UEs' home cells is denoted by $\mathcal{C}$. Let $\mathcal{J}_{i}^{-}=\left\{j: c_{j}=i\right\}$, i.e., the set of UEs of which the corresponding home cell is $i$.

We allow JT in our model, so any UE $j$ can be served by multiple cells simultaneously. For the sake of presenting the system model, consider a (generic) cell-UE association solution, for which the set of cells serving UE $j$ and the set of UEs served by cell $i$ are denoted by $\mathcal{J}_{j}$ and $\mathcal{J}_{i}$, respectively. Note that $i \in \mathcal{J}_{j} \Leftrightarrow j \in \mathcal{J}_{i}$. For the sake of presentation, we selectively use either according to the context. Note that we have $\mathcal{J}_{j} \subseteq \mathcal{J}_{j}^{+}$and $\mathcal{J}_{i} \subseteq \mathcal{J}_{i}^{+}$for any $j \in \mathcal{J}$ and $i \in \mathcal{J}$, respectively. For each UE $j$, we use the notation $d_{j}$ to denote its bit rate demand. For any $\mathfrak{j} \in \mathcal{J}$, we assume $c_{\mathfrak{j}} \in \mathcal{J}_{\mathfrak{j}}$. Thus $\mathcal{J}_{i}^{-} \subseteq \mathcal{J}_{i}$ holds for any $i \in \mathcal{J}$.

\section{B. Load Coupling}

For this moment, we fix the cell-UE association. Without loss of generality, we use RU as the minimum unit for resource allocation, composed by one or more resource blocks in orthogonal frequency division multiple access (OFDMA).

$$
\begin{aligned}
\gamma_{j} \triangleq h_{j}(x) & =\frac{\sum_{i \in \mathcal{J}_{j}} p_{i} g_{i j}}{\sum_{k \in \mathcal{J} \backslash \mathcal{J}_{j}} p_{k} g_{k j} x_{k}+\sigma^{2}} \\
\gamma & \triangleq\left[\gamma_{1}, \gamma_{2}, \ldots, \gamma_{m}\right]
\end{aligned}
$$

Eq. (1) models the SINR at UE $j \in \mathcal{J}$. The network-wide SINRs are represented by the vector shown in Eq. (2). In Eq. (1), $p_{i}\left(p_{i}>0\right)$ denotes the transmit power per RU (in time and frequency) of cell $i$. Notation $g_{i j}$ is the power gain between cell $i$ and UE $j$. The summation $\sum_{i \in \mathcal{J}_{j}} p_{i} g_{i j}$ is the received signal power at UE $j$ via $J T$, from all the UE $j$ 's serving cells in $\mathcal{J}_{j}$. In the denominator, $\sigma^{2}$ is the noise power. Entity $x_{k}$ is the load of cell $k$, which is defined to be the proportion of RUs consumed in cell $k$ for all UEs in $\partial_{k}$. We model the interference that UE $j$ receives from other cells by the term $\sum_{k \in \mathcal{J} \backslash \mathcal{J}_{j}} p_{k} g_{k j} x_{k}$. For any RU in cell $i, x_{k}$ is intuitively interpreted as the likelihood that the served UEs of cell $i$ receive the interference from the cell $k$. The SINR of any UE $j$ is a function of the load vector $x$, denoted by $h_{j}(x)$.

$$
\begin{aligned}
x_{i} \triangleq f_{i}(\gamma) & =\sum_{j \in \mathcal{J}_{i}} \frac{d_{j}}{M B \log _{2}\left(1+\gamma_{j}\right)} \\
x & \triangleq\left[x_{1}, x_{2}, \ldots, x_{n}\right]
\end{aligned}
$$

The load of any cell $i$, is represented in Eq. (3), so as to satisfy the bit rate demands of its served UEs. Eq. (4) gives the network-wide cell load. In the denominator, B is the bandwidth per RU and $M$ is the total number of RUs available. The entity $B \log _{2}\left(1+\gamma_{j}\right)$ is the achievable bit rate per RU. Thus, $M B \log _{2}\left(1+\gamma_{j}\right)$ computes the total achievable bit rate for $U E j$. The term $d_{j} / M B \log _{2}\left(1+\gamma_{j}\right)$ computes the required amount of RUs to satisfy the demand $d_{j}$. Then $x_{i}=\sum_{j \in \mathcal{J}_{i}} d_{j} / M B \log _{2}\left(1+\gamma_{j}\right)$ is the proportion of the RUs consumed for transmission in cell $i$, which complies to the definition of the cell load. It can be verified in Eq. (3), that if any UE $j$ satisfies $j \in \mathcal{J}_{i} \cap \mathcal{J}_{k}(k \neq i)$ (meaning that $j$ is currently served by cell $i$ and $k$ via JT), then the number of RUs consumed by UE $j$ in both cells $i$ and $k$ are equal. The load of cell $i$ is a function of the SINRs $\gamma_{j}$ for all $j \in \mathcal{J}_{i}$, denoted by $f_{i}(\gamma)$ in Eq. (3).

We have the coupling equations for both UE's SINR and cell's load, shown in Eq. (5).

$$
\text { load-coupling: }\left\{\begin{array}{l}
\gamma=\mathbf{h}(\boldsymbol{x}) \\
\boldsymbol{x}=\mathbf{f}(\boldsymbol{\gamma})
\end{array}\right.
$$

Lemma 1 shows that a larger $\gamma$ leads to a smaller $\chi$, and vice versa. This can be verified easily in Eq. (1) and Eq. (3).

Lemma 1. The following relationships hold for the coupling equations (5).

1) $\mathbf{f}(\gamma) \leqslant \mathbf{f}\left(\gamma^{\prime}\right)$, for $\gamma \geqslant \gamma^{\prime}$.

2) $\mathbf{h}(\boldsymbol{x}) \leqslant \mathbf{h}\left(\boldsymbol{x}^{\prime}\right)$, for $\boldsymbol{x} \geqslant \boldsymbol{x}^{\prime}$.

We remark that the way of modeling interference above is not exact. For two base stations serving a UE with JT, the interference generated to another UE should contain an additional term ${ }^{1}$. One reason of using the approximation is to

\footnotetext{
${ }^{1}$ For cells $i_{1}$ and $i_{2}$ serving jointly a UE, the extra term for the interference to UE $j$ equals $2 \sqrt{p_{i_{1}}} \sqrt{p_{i_{2}}} h_{i_{1} j} h_{i_{2}, j} \cos \left(\theta_{i_{1}}-\theta_{i_{2}}\right)$, where $h_{i_{1} j}$ and $h_{i_{2}, j}$ are the channel gains, and $\theta_{i_{1}}$ and $\theta_{i_{2}}$ are the phases of the two received interfering signals, see [23].
} 
achieve a good trade-off between exactness and complexity, as an exact interference modeling would require the network to acquire and process, for each UE, information related to other individual UEs (rather than information available at the cell level). Moreover, the extra terms incurred due to multiple JT operations tend to offset each other (for a theoretical proof, see [23]). In addition, the approximation is coherent with the load-coupling modeling approach. The approach itself is an approximation of interference (even for the non-JT case), but it suits well (see [3]-[11]) as long as the performance of interest is at an aggregated level with a time scale being greater than that of an RU, which is the case in our study. For these reasons, our way of modeling interference has been used by a number of other authors for JT (e.g., [20], [22], [23]).

\section{Standard Interference Function}

We show that the load-coupling equations can be solved by the fixed-point iteration, based on the fact that both $\mathbf{h}(\mathbf{f}(\boldsymbol{\gamma}))$ and $\mathbf{f}(\mathbf{h}(\boldsymbol{x}))$ are standard interference function (SIF).

Definition 1. A function $\vartheta: \mathbb{R}_{+}^{\mathrm{m}} \rightarrow \mathbb{R}_{++}$is called an SIF if the following properties hold:

1) (Scalability) $\alpha \vartheta(\mu)>\vartheta(\alpha \mu), \mu \in \mathbb{R}_{+}^{m}, \alpha>1$.

2) (Monotonicity) $\vartheta(\boldsymbol{\mu}) \geqslant \vartheta\left(\boldsymbol{\mu}^{\prime}\right)$, if $\boldsymbol{\mu} \geqslant \boldsymbol{\mu}^{\prime}$.

Property 1. Suppose the function $\vartheta$ is an SIF. For the sequence $\boldsymbol{\mu}^{(0)}, \boldsymbol{\mu}^{(1)}, \ldots$ generated by fixed-point iterations, if there exists $k$ satisfying $\vartheta\left(\mu^{(k)}\right) \leqslant \vartheta\left(\mu^{(k-1)}\right)$, then the sequence $\mu^{(k)}, \mu^{(k+1)}, \ldots$ is monotonously decreasing (in every component), and converges to a unique fixed point.

Property 2. Suppose $A \in \mathbb{R}^{n \times m}, \mu \in \mathbb{R}^{m}$ and $\mathbf{b} \in \mathbb{R}^{n}$. Define $\varphi(\boldsymbol{\mu}): \mathbb{R}_{+}^{\mathrm{n}} \rightarrow \mathbb{R}$. If $\varphi_{1}$ is concave in $\boldsymbol{\mu}$, so is $\varphi(\boldsymbol{A} \boldsymbol{\mu}+$ b).

Property 3. Denote $\varphi_{1}: \mathbb{R}^{k} \rightarrow \mathbb{R}$. Denote $\varphi_{2}: \mathbb{R}^{n} \rightarrow \mathbb{R}^{k}$. Then the function $\varphi_{1}\left(\varphi_{2}(\cdot)\right)$ is concave, if $\varphi_{1}$ is concave and nondecreasing, and $\varphi_{2}$ is concave.

Lemma 2. The two functions $\mathbf{f}(\mathbf{h}(\boldsymbol{x}))$ and $\mathbf{h}(\mathbf{f}(\boldsymbol{\gamma}))$ are SIF.

Proof. The monotonicity is shown by Lemma 1. To prove the scalability, we first show the concavity of $\mathbf{h}(\mathbf{f}(\boldsymbol{\gamma}))$ in $\boldsymbol{\gamma}$. Define function $\zeta$ as $\zeta(\varphi) \triangleq \sum_{i \in \mathcal{J}_{j}} p_{i} g_{i j} /\left(\sum_{k \in \mathcal{J} \backslash \mathcal{J}_{j}} p_{k} g_{k j} \sum_{j \in \mathcal{J}_{i}} \frac{r_{j}}{\varphi_{j}}+\sigma^{2}\right)$. Note that function $\mathbb{R} \rightarrow \mathbb{R}: \chi \mapsto 1 /\left(\frac{1}{\chi}+1\right)$ is concave. Combined with Property $2, \zeta$ is concave. Define the function $\varphi_{j}$ as $\varphi_{j}\left(\gamma_{j}\right) \triangleq \log \left(1+\gamma_{j}\right)$. Note that function $\mathbb{R} \rightarrow \mathbb{R}: \chi \mapsto \log _{2}(1+\chi)$ is concave. According to Property $2, \varphi_{j}$ is concave in $\gamma_{j}$. We remark that $\zeta$ is concave and nondecreasing. By Property $3, h_{j}=\zeta(\varphi(\gamma))$ is concave in $\gamma$. We then show $\mathbf{f}(\mathbf{h}(\boldsymbol{x}))$ is concave in $\boldsymbol{x}$. Note that function $\mathbb{R} \rightarrow \mathbb{R}: \chi \mapsto 1 \log \left[1 /\left(1+\frac{1}{x}\right)\right]$ is concave, thus for any $i \in[1, n], f_{i}(h(x))$ is concave, according to Property 2 . By the conclusion in [8] that the scalability holds for any strictly concave function. Hence the conclusion.

By Lemma 2 and Property 1, there exists a unique fixed point for function $\mathbf{f}(\mathbf{h}(\boldsymbol{x}))$. The same conclusion applies for function $\mathbf{h}(\mathbf{f}(\boldsymbol{\gamma}))$.
Lemma 3. Suppose $\tilde{\boldsymbol{x}}$ is the fixed point of function $\mathbf{f}(\mathbf{h}(\mathbf{x}))$, i.e., $\tilde{\boldsymbol{\chi}}$ satisfies $\tilde{\boldsymbol{\chi}}=\mathbf{f}(\mathbf{h}(\tilde{\mathbf{x}}))$, and let $\tilde{\gamma}=\mathbf{h}(\tilde{\boldsymbol{x}})$. Then $\tilde{\gamma}$ is the fixed point of function $\mathbf{h}(\mathbf{f}(\boldsymbol{\gamma}))$, i.e., $\tilde{\boldsymbol{\gamma}}=\mathbf{h}(\mathbf{f}(\tilde{\boldsymbol{\gamma}}))$ holds for $\tilde{\boldsymbol{\gamma}}$, and vice versa.

Proof. Since $\tilde{\boldsymbol{x}}=\mathbf{f}(\tilde{\gamma})$, by $\tilde{\gamma}=\mathbf{h}(\tilde{\mathbf{x}})$, we get $\tilde{\gamma}=\mathbf{h}(\mathbf{f}(\tilde{\gamma}))$.

\section{Problem Formulation AND COMPleXity ANALYSIS}

\section{A. Formulation}

The two problems MinSumL and MinMaxL are formulated in this section. Here, the association is represented by an $n \times m$ matrix $\kappa$, and $\kappa_{i j}=1$ if and only if cell $i$ is currently serving $\mathrm{UE} j$.

$$
\begin{aligned}
& {[\text { MinSumL }] \quad \min _{\kappa} \sum_{i \in \mathcal{J}} x_{i}} \\
& \text { s.t. } \quad x=\mathbf{f}(\mathbf{h}(\boldsymbol{x}, \boldsymbol{\kappa}), \boldsymbol{\kappa}) \\
& 0<x_{i} \leqslant 1 \quad i \in \mathcal{J} \\
& K_{i j}=0 \quad i \notin \mathcal{J}_{j}^{+}, j \in \mathcal{J} \\
& \kappa_{i j}=1 \quad i \in \mathcal{C}, j \in \mathcal{J}_{i}^{-} \\
& K_{i j} \in\{0,1\} \quad i \in \mathcal{J}, j \in \mathcal{J} \\
& \begin{aligned}
{[\text { MinMaxL }] } & \min _{\kappa} \max _{i \in \mathcal{J}} x_{i} \\
\text { s.t. } & \text { Eq. (6b) - Eq. (6f) }
\end{aligned}
\end{aligned}
$$

The optimization variable in both two problems is $\kappa$. In MinSumL, load-coupling constraints are shown in Eq. (6b). Eq. (6c) guarantees that the cell load value is positive but less or equal than 1 . The variable $\kappa$ is binary. Eq. (6d) imposes that $\mathcal{J}_{j} \subseteq \mathcal{J}_{j}^{+}$and $\mathcal{J}_{i} \subseteq \mathcal{J}_{i}^{+}, i \in \mathcal{J}$. Constraint Eq. (6e) imposes $\mathcal{J}_{i}^{-} \subseteq \mathcal{J}_{i}, i \in \mathcal{J}$. MinMaxL differs only in the objective with MinSumL.

\section{B. Complexity Analysis}

Theorem 4. Both MinSumL and MinMaxL are NP-hard.

Proof. The idea is to use a polynomial-time reduction from the 3-satisfiability (3-SAT) problem that is $\mathcal{N} \mathcal{P}$-complete. Consider a 3-SAT instance with $\mathrm{n}$ Boolean variables $\mathrm{b}_{1}, \mathrm{~b}_{2}, \ldots, \mathrm{b}_{\mathrm{n}}$, and $m$ clauses. A variable or its negation is referred to as a literal. Denote by $\hat{b}_{i}$ the negation of $b_{i}, i \in[1, n]$. Each clause is composed by a disjunction of exactly three distinct literals, e.g., $\left(b_{1} \vee b_{2} \vee b_{n}\right)$. The 3-SAT problem amounts to determining whether or not there exists an assignment of true/false values to the variables, such that all clauses are satisfied (i.e., at least one literal has value true in every clause). For any instance of 3-SAT, we construct a corresponding network scenario shown in Figure 1. The transmit power of all cells, the demand of all UEs, and the noise effect $\sigma^{2}$ are all uniformly set to 1.0 . For all variables $b_{1}, b_{2}, \ldots, b_{n}$, we define $n$ UEs $u_{1}, u_{2}, \ldots, u_{n}$. For the $m$ clauses, we define UEs $u_{n+1}, u_{n+2}, \ldots, u_{n+m}$. For any $u_{i}, i \in[1, n+m]$, there is a home cell $c_{i}$. According to the system model in Section II, $u_{i}$ is associated with $c_{i}$, and possibly other cells. For any 


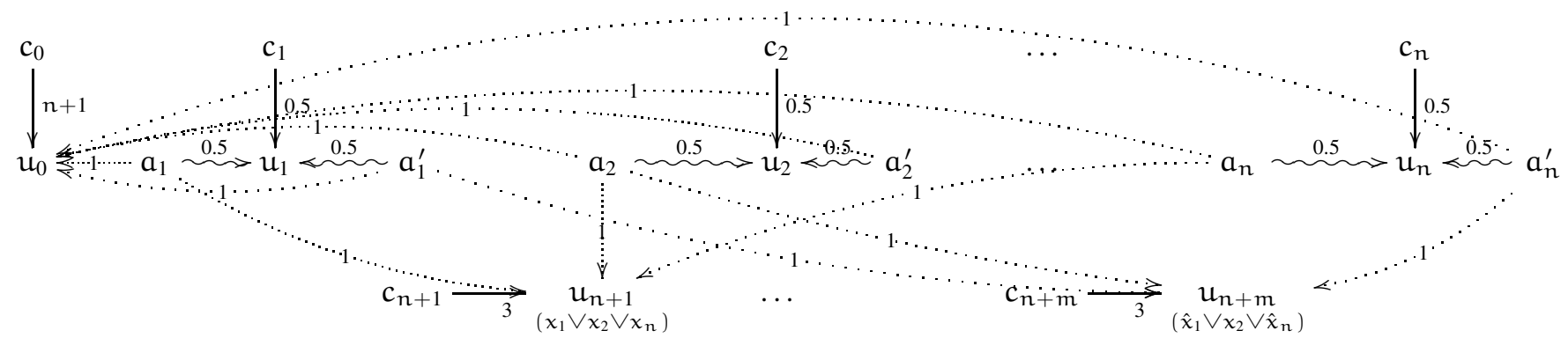

Figure 1. The instance for $\mathcal{N} \mathcal{P}$-hardness reduction. There are $n+m+1$ UEs in total, denoted by $u_{0}, u_{1}, \ldots, u_{n+m}$, respectively. For each $i \in[0, n+m]$, the home cell of $U E u_{i}$ is $c_{i}$. For any $k \in[n+1, n+m], u_{k}$ corresponds to a clause. The numbers on the lines with arrows are the power gains of UEs. The gains not shown are negligible and treated as zero.

variable $b_{i}$, we define two cells $a_{i}$ and $a_{i}^{\prime}$, representing the two literals and having symmetric gain for UE $u_{i}$. There are thus $3 n+m$ cells in total. The gain values between the cells and UEs are shown in Figure 1. For any $U E u_{i}, i \in[1, n+m]$, the gain values of $c_{i}, a_{i}$ and $a_{i}^{\prime}$ equal 0.5 . For any $i \in[1, \mathrm{~m}]$, UE $u_{n+i}$ has gain 1.0 from the cells that represent the literals in clause $i$, whereas the gains from the other cells are negligible. For example, the last clause in Figure 1, i.e., clause $m$, is $\left(\hat{b}_{1} \vee b_{2} \vee \hat{b}_{n}\right)$, defined for UE $u_{n+m}$. Then $u_{n+m}$ has nonnegligible gain from $a_{1}^{\prime}, a_{2}$ and $a_{n}^{\prime}$, and these gains are set to 1.0. In addition, we define an extra cell-UE pair $c_{0}$ and $u_{0}$. The gain values from $a_{i}, a_{i}^{\prime}, i \in 1, n$ to $u_{0}$ are 1.0. All other gains are negligible, treated as zero and not shown in Figure 1.

We make several observations. For any $i \in[1, n]$, if $u_{i}$ is only served by the home cell $c_{i}$, then $\gamma_{u_{i}}=0.5 / 1=$ 0.5 implying that $x_{\mathfrak{c}_{i}}=1 / \log _{2}(1+0.5)>1$ and hence the demand of $u_{i}$ cannot be satisfied. For any $i \in[1, n]$, if $u_{i}$ is served by both $a_{i}$ and $a_{i}^{\prime}$ besides the home cell $c_{i}$, then $c_{0}$ will be overloaded. To arrive at the conclusion, observe that $\gamma_{\mathfrak{u}_{i}}=$ $(0.5+0.5+0.5) / 1=1.5$ resulting in $x_{a_{i}}=x_{a_{i}^{\prime}}=\log _{2} 2.5$. Then $\gamma_{u_{0}}=(n+1) /\left(2 \log _{2} 2.5+(n-1)+1\right)<1$, leading to $x_{c_{0}}>1$. Therefore the demand of $u_{0}$ cannot be met. Hence, for any pair $a_{i}$ and $a_{i}^{\prime}$, one and exactly one of them will have UE associated, and its load is $1 / \log _{2}(1+(0.5+0.5) / 1)=1.0$. For each clause, the three cells corresponding to the literals of the clause cannot be all active in serving UEs. Consider for example clause $\left(b_{1} \vee b_{2} \vee b_{n}\right)$. If $a_{1}, a_{2}$, and $a_{n}$ are all serving UEs, then $\gamma_{u_{n+1}}=3 /(1 \times 1.0+1 \times 1.0+1 \times 1.0+1)<1$, causing $x_{\mathfrak{c}_{n+1}}>1$. However, we can verify that the demand of $u_{n+1}$ can be satisfied if at most two of $a_{1}, a_{2}$ and $a_{n}$ have UEs to serve.

Suppose there is an association that ensures all the user's demands satisfied. For each variable $b_{i}$, we set its value to be true if $a_{i}^{\prime}$ is serving any UE. Otherwise $a_{i}$ must be serving UEs instead, and we set $b_{i}$ to be false. Now we evaluate the satisfiability of each clause. For convenience, consider clause $\left(\hat{x}_{1} \vee x_{2} \vee \hat{x}_{n}\right)$ in Figure 1. Since not all three cells $a_{1}^{\prime}, a_{2}$ and $a_{n}^{\prime}$ can be in the status of serving UEs, at least one of $\hat{b}_{1}, b_{2}$ and $\hat{b}_{n}$ is true, and the clause is true. Thus the 3-SAT instance is feasible. Conversely, suppose we have a feasible solution for the 3-SAT instance. Then we choose $a_{i}$ to serve, together with $c_{i}$, UE $u_{i}$, if $\hat{b}_{i}$ is true. If $\hat{b}_{i}$ is false, $a_{i}^{\prime}$ is chosen instead. Doing so satisfies the demands $\mathfrak{u}_{0}, \mathfrak{u}_{1}, \ldots, \mathfrak{u}_{n}$. Moreover, the demands $u_{n+1}, u_{n+2}, \ldots, u_{n+m}$ become satisfied as well,

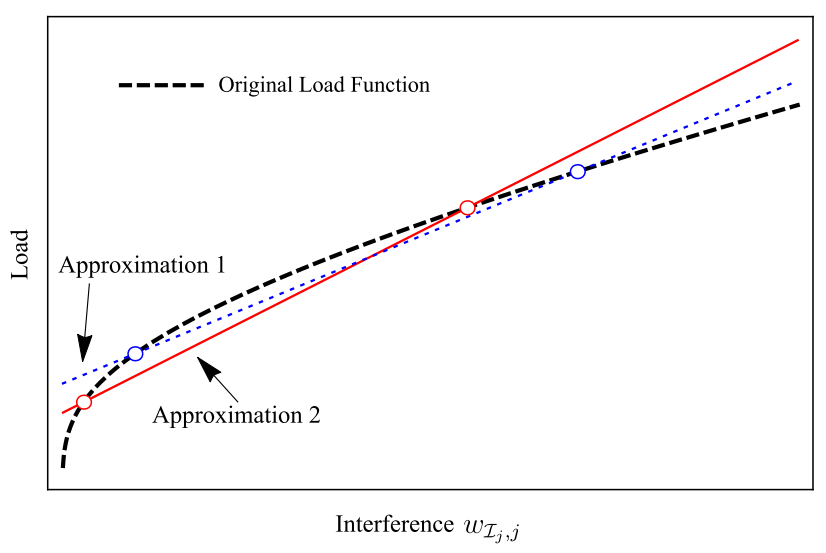

Figure 2. Linear approximations for the concave load-coupling function. The horizontal axis is the received interference and the vertical axis is the load for serving a UE. The circles on each line denote the points used for construct the linear approximation.

because at most two out of the three cells defined for the three literals of the clause will be serving any UE. Thus the association is feasible. Hence the conclusion.

Theorem 4 implies that no low-complexity and exact algorithm can be expected, unless $\mathcal{P}=\mathcal{N} \mathcal{P}$. In addition, even for a sub-optimal algorithm, the evaluation of candidate cellUE association solutions is not straightforward, due to the load-coupling constraints (6b) and (7b). For each candidate solution, obtaining the objective value requires to solve the load-coupling equations, and this is typically done by fixedpoint iterations.

\section{Problem Solving Via Linear Approximation}

In this section, we provide a solution approach based on linearization of the load function. We use $f_{\mathcal{J}_{j}, j}$ to refer to the load of serving UE $j$ by JT of cells in $\mathcal{J}_{j}$. Note that this load occurs in all cells in $\mathcal{J}_{\mathfrak{j}}$. The value of $\mathfrak{f}_{\mathcal{J}_{j}, \mathfrak{j}}$ is determined by the SINR, which, in turn, depends on the amount of interference. For UE $j$ served by cells in $\mathcal{J}_{\mathfrak{j}}$, we use $\mathcal{w}_{\mathcal{J}_{j}, j}$ to denote the interference variable, i.e., $w_{\mathcal{J}_{j}, j}=\sum_{k \in \mathcal{J} \backslash \mathcal{J}_{j}} p_{k} g_{k j} x_{k}$. It is easily proved that function $f_{\mathcal{J}_{j}, j}$ is concave in $\mathcal{W}_{\mathfrak{J}_{j}, j}$ (cf. Lemma 2), see Figure 2 for an illustration. The load-coupling equation is then as follows. 


$$
\begin{cases}x_{i}=\sum_{j \in \mathcal{J}_{i}} f_{\mathcal{J}_{j}, j}\left(w_{\mathcal{J}_{j}, j}\right) & i \in \mathcal{J} \\ w_{\mathcal{J}_{j}, j}=\sum_{k \in \mathcal{J} \backslash \mathcal{J}_{j}} p_{k} g_{k j} x_{k} & j \in \mathcal{J}\end{cases}
$$

Consider replacing $f\left(\mathcal{w}_{\mathcal{J}_{j}, j}\right)$ in Eq. (8) by a linear function as an approximation. The linear approximation can be obtained by taking two points within the interference range of interest and evaluating the nonlinear load function values, followed by constructing the corresponding linear function that coincides with the nonlinear function at these two points. Denote the two points by $W_{o} \mathcal{J}_{j}, j$ and ${\stackrel{\circ}{\mathcal{J}_{j}, j}}_{j}$, with $W_{\mathcal{J}_{j}, j}<{\stackrel{\circ}{\mathcal{J}_{j}, j}}_{j}$, for UE $j$ and its serving cell set $\mathcal{J}_{j}$. The approximation is illustrated in Figure 2, for two different choices of $W_{\mathcal{J}_{j}, j}$ and $\mathfrak{W}_{\mathcal{J}_{j}, j}$. In the figure, the $x$-axis is the interference variable $\mathcal{w}_{\mathcal{J}_{j}, j}$, of which the magnitude is typically $10^{-10}$ for a UE. We do not explicitly show the values of the axis to keep the generality of the illustration, because the interference depends highly on the specific scenario and UE under consideration.

In equation form, the linear approximation reads

$$
l_{\mathcal{J}_{j}, j}\left(w_{\mathcal{J}_{j}, j}\right) \triangleq s_{\mathcal{J}_{j}, j} \mathcal{w}_{\mathcal{J}_{j}, j}+\mu_{\mathcal{J}_{j}, j}
$$

where the two constants $s_{\mathcal{J}_{j}, j}$ and $\mu_{\mathcal{J}_{j}, j}$ are computed as follows.

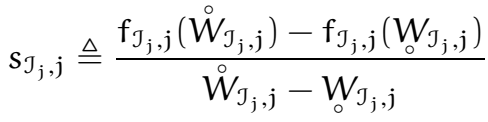

$$
\begin{aligned}
& \mu_{\mathcal{J}_{j}, j} \triangleq f_{\mathcal{J}_{j}, j}\left(W_{o} \mathcal{J}_{j}, j\right)-W_{o} \mathcal{J}_{j}, j s_{\mathcal{J}_{j}, j}
\end{aligned}
$$

We use $\mathcal{L}_{j}$ to denote the set of possible association scenarios for UE $j$; each element of $\mathcal{L}_{j}$ is a subset of the candidate set $\mathcal{J}_{j}^{+}$. Note that $\left|\mathcal{L}_{j}\right|=2^{\left|\mathcal{J}_{j}^{+}\right|-1}$. This is because by minimum one cell has to be selected for association, and the association must contain at least the home cell $c_{j}$ (which is an element of $\mathcal{J}_{j}^{+}$). In the sequel, we use $\ell$ to index the elements of $\mathcal{L}_{j}$. Note that because $\mathcal{L}_{j}$ is a set of sets, $\ell \in \mathcal{L}_{j}$ is a set and corresponds to entity $\mathcal{J}_{j}$ in the above introduction of linear approximation. The association variable $\kappa_{i j}$ in MinSumL is accordingly generalized to be $\kappa_{\ell, j}$. We further introduce a constant $T_{\ell, j}$, defined as

$$
\mathrm{T}_{\ell, j} \triangleq \sum_{i \in \mathcal{J} \backslash \ell} p_{i} g_{i j}, \quad \ell \in \mathcal{L}_{j}, j \in \mathcal{J}
$$

Note that $T_{\ell, j}$, by definition, is an upper bound of interference, i.e., $w_{\ell, j} \leqslant T_{\ell, j}, \ell \in \mathcal{L}_{j}, j \in \mathcal{J}$, because maximum load of all interfering cells is assumed in (12).

Based on the linear approximation, we derive an MILP formulation of MinSumL. The MILP formulation is presented below.

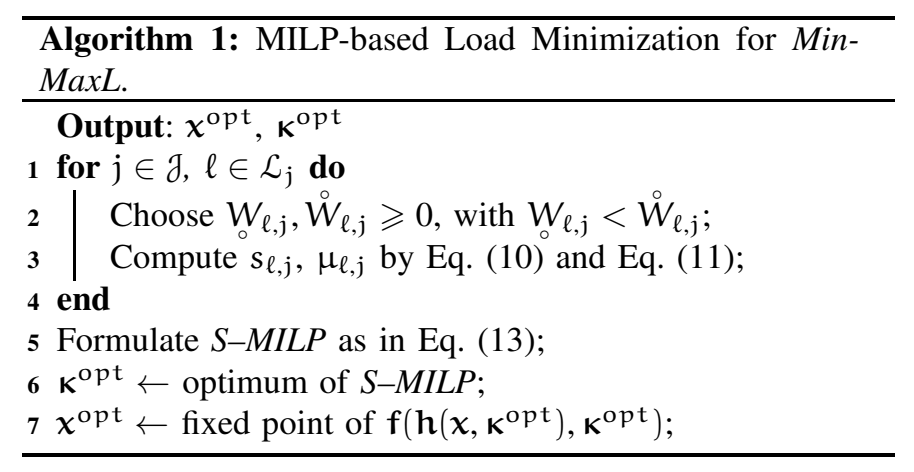

$$
\begin{array}{ll}
{[S-M I L P]} & \min _{\mathcal{w}, \mathrm{k}} \sum_{i \in \mathcal{J}} x_{i} \\
\text { s.t. } x_{i}=\sum_{j \in \mathcal{J}} \sum_{\ell \in \mathcal{L}_{j}: i \in \ell}\left(s_{\ell, j} w_{\ell, j}+\mu_{\ell, j} \kappa_{\ell, j}\right) & i \in \mathcal{J} \\
w_{\ell, j} \geqslant \sum_{i \in \mathcal{J} \backslash \ell} p_{i} g_{i j} x_{i}-T_{\ell, j}\left(1-\kappa_{\ell, j}\right) & \ell \in \mathcal{L}_{j}, j \in \mathcal{J} \\
w_{\ell, j} \geqslant 0 & \ell \in \mathcal{L}_{j}, j \in \mathcal{J} \\
0 \leqslant x_{i} \leqslant 1 & i \in \mathcal{J} \\
\sum_{\ell \in \mathcal{L}_{j}} \kappa_{\ell, j}=1 & \\
\kappa_{\ell, j} \in\{0,1\} & \ell \in \mathcal{J}
\end{array}
$$

Selecting one $\kappa_{\ell, j}\left(\ell \in \mathcal{L}_{j}\right)$, as done in Eq. (13f), corresponds to Eq. (6d) and Eq. (6e). The constraints shown in Eq. (13b)-Eq. (13d) provide the effect of a linear approximation of the non-linear constraint (6b). Note that the desired term in the right-hand side of Eq. (13c) is $\left(s_{\ell, j} w_{\ell, j}+\mu_{\ell, j}\right) \kappa_{\ell, j}$, which is nonlinear because $\boldsymbol{w}_{\ell, j}$ and $\kappa_{\ell, j}$ are both variables. To see that we can achieve the same effect using $\left(s_{\ell, j} w_{\ell, j}+\mu_{\ell, j} \kappa_{\ell, j}\right)$ and the other constraints, consider separately the two cases $\kappa_{\ell, j}=0$ and $\kappa_{\ell, j}=1$. If $\kappa_{\ell, j}=0$, Eq. (13c) becomes void, and hence there is no restriction on $w_{\ell, j}$ except non-negativity. Due to minimization, $\boldsymbol{w}_{\ell, j}=0$, and hence the entire term $s_{\ell, j} w_{\ell, j}+\mu_{\ell, j} \kappa_{\ell, j}$ equals 0 , meaning that $j$ does not impose any load of cells not serving the UE. If $\kappa_{\ell, j}=$ 1 , then clearly $\left(s_{\ell, j} w_{\ell, j}+\mu_{\ell, j} \kappa_{\ell, j}\right)=\left(s_{\ell, j} w_{\ell, j}+\mu_{\ell, j}\right) \kappa_{\ell, j}$. In this case, UE $j$ is associated with cells in $\ell$, and $w_{\ell, j} \geqslant$ $\max \left\{\sum_{i \in \mathcal{J} \backslash \ell} p_{i} g_{i j} x_{i}, 0\right\}$ by Eq. (13c) and Eq. (13d). Since the problem is minimization, either Eq. (13c) or Eq. (13d) will hold with equality. Thus we have $w_{\ell, j}=\sum_{i \in \mathcal{J} \backslash \ell} p_{i} g_{i j} x_{i}$ that indeed is the total amount of interference, provided that the set of serving cells is $\ell$. Consequently the term $s_{\ell, j} \mathcal{w}_{\ell, j}+\mu_{\ell, j} \kappa_{\ell, j}$ represents the load of the serving cells for $\mathrm{UE} j$, computed by the linear function. For MinMaxL, the corresponding MILP formulation can be obtained via a slight modification of Eq. (13a). We omit further details as the modification is straightforward. The solution approach of using the MILP formulation for MinSumL is summarized in Algorithm 1. 
The main advantage of using the linear approximation is to enable a linear form of the optimization problem as an MILP, for which there are standard optimization tools for problem solving to approach global optimum. This serves two purposes. First, it yields an approximative solution to the original problem, enabling a vis-a-vis comparison to any algorithm that focuses on low complexity. Such an algorithm is presented later in Section V. Second, and more importantly, we will demonstrate how problem solving using linear approximation leads to a bounding scheme, in terms of providing a lower bound on the global minimum that effectively gauges the amount of (worst-case) optimality gap and thereby performance assessment of any sub-optimal algorithm. To these ends, Algorithm 1 is not distributed by nature. Rather, it is intended for use within the cloud radio access network (CRAN) architecture for future networks.

We remark that for solving $S-M I L P$, the standard approach consists in branch-and-bound (and branch-and-cut), which scales substantially better than an exhaustive search. Still, the scalability is an issue, as the complexity, in the worst-case, is exponential; this justifies the derivation of the algorithm in Section V. Note also that, for the purpose of bounding to gain performance insights of other, low-complexity algorithms, Algorithm 1 is not intended to be run online.

Clearly, the solution from solving the linear optimization problem varies by the chosen linear approximation which is defined by the points $W_{\mathcal{J}_{j}, j}$ and ${\stackrel{W}{\mathcal{J}_{j}, j}}_{j}$. In general, these points should be close to the amount of interference $\mathcal{w}_{\mathcal{J}_{j}, j}$ at global optimum, which is of course not known a priori. However, if one has a solution from any sub-optimal algorithm, e.g., the algorithm we derive in Section $\mathrm{V}$, the output (i.e., expected interference) can be used to guide the selection. Moreover, if $W_{\mathcal{J}_{j}, j}$ and ${\stackrel{\circ}{\mathcal{J}_{j}, j}}_{j}$ are chosen such that the interference at optimum falls within the range $\left[W_{\mathcal{J}_{j}, j},{\stackrel{\circ}{\mathcal{J}_{j}, j}}_{j}\right]$, then solving the problem using the approximation guarantees a lower bound of the global minimum. The trivial choice to achieve the bounding effect is to set $W_{o} \mathcal{J}_{j}, j$ and $\stackrel{\circ}{W}_{\mathcal{J}_{j}, j}$ to zero and the most extreme of amount interference, respectively. The latter corresponds to assuming all other cells are interfering with full load. However, as can be realized from Figure 2, the gap between the linear function and the nonlinear one can be large for this choice of range. Later in Section VI, we derive nontrivial yet tractable solutions to reduce the range (while still guaranteeing its validity) and thereby significantly strengthen the bound.

Instead of using the type of linear approximation that has been discussed above, one can consider the first-order Taylor series approximation, by constructing a linear function via the first-order derivative of the nonlinear function at a selected point, such as the middle point of $\left[W_{\mathcal{J}_{j}, j},{\stackrel{W}{\mathcal{J}_{j}, j}}_{j}\right]$. This linear approximation, although can be used in the MILP formulation for a problem solution, does not enable a bounding scheme, because the approximation over-estimates the true load value. We also remark that higher orders of Taylor series approximation result in nonlinear objective functions that, from an optimization viewpoint, do not exhibit advantage in comparison to the original nonlinear function.

\section{LOAD MinimiZATION VIA OPTIMALITY CONDITIONS}

The second solution approach that we propose is based on improving cell load by adjusting cell-UE association. This approach can be used in conjunction with the MILPbased solutions, to further improve the association obtained by solving the MILPs. For a UE and its serving cells, an adjustment amounts to either expanding the set of serving cells with one additional cell, or removing one cell from the set of serving cells. In the sequel, we use the term link adjustment to refer to the action of updating the cell-UE association as described above.

\section{A. Link Adjustment: Basic Properties}

For link adjustment, we use $v$ and $u$ to respectively denote the cell and UE under consideration. The corresponding load and the SINR functions are $f_{v}(\boldsymbol{x})$ and $h_{u}(\boldsymbol{\gamma})$, as defined in Eq. (1) and Eq. (3), with the only notational difference that $v$ and $u$ replace $i$ and $j$, respectively, and hence the set of serving cells in Eq. (1) is $\mathcal{J}_{\mathfrak{u}}$, and the set of served UEs in Eq. (3) is $\partial_{v}$.

Suppose $v \notin \mathcal{J}_{\mathfrak{u}}$, and the link adjustment operation is to expand the set of serving cells to $\mathcal{J}_{\mathfrak{u}} \cup\{v\}$. Consequently the set of cells of $U E u$ is reduced from $\mathcal{J} \backslash \mathcal{J}_{\mathfrak{u}}$ to $\mathcal{J} \backslash\left(\mathcal{J}_{\mathfrak{u}} \cup\{v\}\right)$. We denote the vector of SINR functions, resulted from the link adjustment, by $\mathbf{h}^{+}$, with $\mathbf{h}^{+}=\left[h_{1}^{+}(\boldsymbol{x}), h_{2}^{+}(\boldsymbol{x}), \ldots, h_{m}^{+}(\boldsymbol{x})\right]$, where for UE $u, h_{u}^{+}$is given in Eq. (14), and $h_{j}^{+}(x)=h_{j}(x)$, for all $j \neq u$, because the cell association remains for UEs other than $u$.

$$
h_{u}^{+}(x) \triangleq \frac{\sum_{i \in \mathcal{J}_{u} \cup\{v\}} p_{i} g_{i u}}{\sum_{k \in \mathcal{J} \backslash\left(\mathcal{J}_{j} \cup\{v\}\right)} p_{k} g_{k u} x_{k u}+\sigma^{2}}
$$

For cell $v$, the set of associated UEs is expanded from $\partial_{v}$ to $\mathcal{J}_{v} \cup\{u\}$. For all cells other than $v$, the UE association remains. Thus the vector of load function, after the new, augmented association of UE $u$ to cell $v$, is $\mathbf{f}^{+}(\gamma)=$ $\left[f_{1}^{1}(\gamma), f_{2}^{+}(\gamma), \ldots, f_{n}^{+}(\gamma)\right]$, where for cell $v$ the load function $f_{v}^{+}$is formulated in Eq. (15), and $f_{i}^{+}(x)=f_{i}(x)$, for all $i \neq v$.

$$
\mathrm{f}_{v}^{+}(\gamma) \triangleq \sum_{j \in \mathcal{J}_{v} \cup\{u\}} \frac{r_{j}}{M B \log _{2}\left(1+\gamma_{u}\right)}
$$

Next, we define the corresponding entities for removing one existing cell-UE association of UE $u$ and cell $v$, assuming that $v$ is not the home cell of UE $u$. That is, the set of UE $u$ 's serving cells is reduced from $\mathcal{J}_{\mathfrak{u}}$ to $\mathcal{J}_{\mathfrak{u}} \backslash\{v\}$. The set of cells generating interference to $u$ is expanded from $\mathcal{J} \backslash \mathcal{J} u$ to $\left(\mathcal{J} \backslash \mathcal{J}_{\mathfrak{u}}\right) \cup\{v\}$, and the set of UEs associated with cell $v$ is reduced from $\partial_{v}$ to $\partial_{v} \backslash\{u\}$. The resulting SINR and load functions are shown in Eq. (16) and Eq. (17), and denoted by $h_{u}^{-}$and $f_{v}^{-}$, respectively. The two vectors of functions are consistently denoted by $\mathbf{h}^{-}$and $\mathbf{f}^{-}$. Note that for any cell $i \neq v, f_{i}^{-}(\gamma)=f_{i}(\gamma)$, and for any UE $j \neq u, h_{j}^{-}(x)=h_{j}(x)$.

$$
\begin{aligned}
& h_{u}^{-}(x) \triangleq \frac{\sum_{i \in \mathcal{J}_{u} \backslash\{v\}} p_{i} g_{i u}}{\sum_{k \in\left(\mathcal{J} \backslash \mathcal{J}_{u}\right) \cup\{v\}} p_{k} g_{k u} x_{k u}+\sigma^{2}} \\
& f_{v}^{-}(\gamma) \triangleq \sum_{j \in \mathcal{J}_{v} \backslash\{u\}} \frac{r_{j}}{M B \log _{2}\left(1+\gamma_{u}\right)}
\end{aligned}
$$


Given the above definitions, the load-coupling equations after expanding respectively reducing one cell-UE association via a link adjustment of cell $v$ and UE $u$, are provided in Eq. (18) and Eq. (19). Here, notation o refers to the compound function, i.e., $\mathbf{f} \circ \mathbf{h}(\cdot)$ means $\mathbf{f}(\mathbf{h}(\cdot))$.

$$
\begin{aligned}
& x=\mathbf{f}^{+} \circ \mathbf{h}^{+}(\boldsymbol{x}) \\
& x=\mathbf{f}^{-} \circ \mathbf{h}^{-}(\boldsymbol{x})
\end{aligned}
$$

We are interested in whether or not the fixed points of above improve the load given by the fixed point of $\mathbf{f} \circ \mathbf{h}(\boldsymbol{x})$. To this end, we derive and prove conditions for load improvement.

Lemma 5. The following properties hold true.

1) $\mathbf{f} \circ \mathbf{h}^{+}(\boldsymbol{x}) \leqslant \min \left\{\mathbf{f} \circ \mathbf{h}(\boldsymbol{x}), \mathbf{f}^{+} \circ \mathbf{h}^{+}(\boldsymbol{x})\right\}, \boldsymbol{x} \in \mathbb{R}_{+}^{n}$.

2) $\mathbf{f} \circ \mathbf{h}^{-}(\boldsymbol{x}) \geqslant \max \left\{\boldsymbol{f} \circ \mathbf{h}(\boldsymbol{x}), \mathbf{f}^{-} \circ \mathbf{h}^{-}(\boldsymbol{x})\right\}, \boldsymbol{x} \in \mathbb{R}_{+}^{n}$.

Proof. For 1), by the monotonicity of $\mathbf{f}$ and that $\mathbf{h}^{+}(\boldsymbol{x}) \geqslant$ $\mathbf{h}(\boldsymbol{x})$ with strict inequality for UE $u$, we obtain $\mathbf{f} \circ \mathbf{h}^{+}(\boldsymbol{x}) \leqslant \mathbf{f} \circ$ $\mathbf{h}(\boldsymbol{x})$. Function $\mathbf{h}$ exhibits monotonicity as well, and $\mathbf{f}^{+}(\boldsymbol{\gamma}) \geqslant$ $\mathbf{f}(\boldsymbol{\gamma})$ for any $\gamma \in \mathbb{R}_{+}^{\mathrm{m}}$, with strict inequality for cell $\nu$, thus $\mathbf{f}^{+} \circ$ $\mathbf{h}^{+}(\boldsymbol{x}) \geqslant \mathbf{f} \circ \mathbf{h}^{+}(\boldsymbol{x})$. Statement 2 ) can be proved analogously.

\section{Lemma 6. The following properties hold true.}

1) $\mathbf{h} \circ \mathbf{f}^{+}(\boldsymbol{\gamma}) \leqslant \min \left\{\mathbf{h} \circ \mathbf{f}(\boldsymbol{\gamma}), \mathbf{h}^{+} \circ \mathbf{f}^{+}(\boldsymbol{\gamma})\right\}, \boldsymbol{\gamma} \in \mathbb{R}_{+}^{m}$.

2) $\mathbf{h} \circ \mathbf{f}^{-}(\boldsymbol{\gamma}) \geqslant \max \left\{\mathbf{h} \circ \mathbf{f}(\boldsymbol{\gamma}), \mathbf{h}^{-} \circ \mathbf{f}^{-}(\boldsymbol{\gamma})\right\}, \boldsymbol{\gamma} \in \mathbb{R}_{+}^{\mathrm{m}}$.

Proof. Because $\boldsymbol{h}$ has the monotonicity property and $\mathrm{f}^{+}(\boldsymbol{\gamma}) \geqslant$ $\mathbf{f}(\boldsymbol{\gamma})$ with strict inequality for cell $\boldsymbol{v}$, we obtain $\mathbf{h} \circ \mathbf{f}^{+}(\boldsymbol{\gamma}) \leqslant$ $\mathbf{h} \circ \mathbf{f}(\boldsymbol{\gamma})$. Utilizing the monotonicity of $\mathbf{f}$ and $\mathbf{h}^{+}(\boldsymbol{x}) \geqslant \mathbf{h}(\boldsymbol{x})$ for any $x \in \mathbb{R}_{+}^{n}$ with strict inequality for UE $u$, we have $\mathbf{h}^{+} \circ \mathbf{f}^{+}(\boldsymbol{\gamma}) \geqslant \mathbf{h} \circ \mathbf{f}^{+}(\boldsymbol{\gamma})$. The observations lead to statement 1 ), and statement 2) can be proved analogously.

\section{B. Conditions for Cell Load Reduction}

We prove several conditions under which the load improves. In Section $\mathrm{V}-\mathrm{C}$, these conditions will be embedded into the link-adjustment optimization algorithm later on.

Definition 2. Given functions $\mathbf{f}, \mathbf{f}^{+}, \mathbf{f}^{-}$and $\mathbf{h}, \mathbf{h}^{+}, \mathbf{h}^{-}$, we define the following notation.

- Denote by $\tilde{\mathbf{x}}$ the fixed point of function $\mathbf{f} \circ \mathbf{h}$, i.e., $\tilde{\mathbf{x}}$ is solution of $\mathbf{x}=\mathbf{f} \circ \mathbf{h}(\boldsymbol{x})$, and denote $\tilde{\gamma} \triangleq \mathbf{h}(\tilde{\mathbf{x}})$.

- Denote by $\underset{\boldsymbol{x}}{+}$ the fixed point of function $\mathbf{f}^{+} \circ \mathbf{h}^{+}$, i.e., $\stackrel{+}{\boldsymbol{x}}$ is the solution of $\mathbf{x}=\mathbf{f}^{+} \circ \mathbf{h}^{+}(\boldsymbol{x})$, and denote $\stackrel{+}{\boldsymbol{\gamma}} \triangleq \mathbf{h}^{+}(\stackrel{+}{\mathbf{x}})$.

- Denote by $\bar{x}$ the fixed point of function $\mathbf{f}^{-} \circ \mathbf{h}^{-}$, i.e., $\bar{x}$ is the solution of $\mathbf{x}=\mathbf{f}^{-} \circ \mathbf{h}^{-}(\boldsymbol{x})$, and denote $\bar{\gamma} \triangleq \mathbf{h}^{-}(\overline{\boldsymbol{x}})$.

Theorem 7. Consider the sequence $\boldsymbol{x}^{(0)}, \boldsymbol{x}^{(1)}, \ldots$, where $\boldsymbol{x}^{(0)}=\tilde{\boldsymbol{x}}$, and $\boldsymbol{x}^{(\mathrm{t})}=\mathbf{f} \circ \mathbf{h}^{+}\left(\boldsymbol{x}^{(\mathrm{t}-1)}\right), \mathrm{t} \geqslant 1$. Then $\boldsymbol{x}^{+} \leqslant \tilde{\boldsymbol{x}}$, if for any iteration $\mathrm{k} \geqslant 1$ we have $\mathrm{f}_{v}^{+} \circ \mathrm{h}^{+}\left(\boldsymbol{x}^{(\mathrm{k})}\right) \leqslant \mathrm{x}_{v}^{(\mathrm{k})}$.

Proof. Suppose $\mathrm{f}_{v}^{+} \circ \mathbf{h}^{+}\left(\boldsymbol{x}^{(k)}\right) \leqslant \boldsymbol{x}_{v}^{(k)}$ for iteration $k$. By Lemma 5, we have $\boldsymbol{x}^{(1)}=\mathbf{f} \circ \mathbf{h}^{+}\left(\boldsymbol{x}^{(0)}\right) \leqslant \mathbf{f} \circ \mathbf{h}\left(\boldsymbol{x}^{(0)}\right)$. Since $\boldsymbol{x}^{(0)}=\tilde{\boldsymbol{x}}, \boldsymbol{x}^{(0)}=\mathbf{f} \circ \mathbf{h}\left(\boldsymbol{x}^{(0)}\right)$ holds. Thus we obtain $\boldsymbol{x}^{(1)} \leqslant \boldsymbol{x}^{(0)}$. Therefore, by Property 1 ,

$$
x^{(k+1)} \leqslant x^{(k)} \leqslant x^{(k-1)} \leqslant \cdots \leqslant x^{(1)} \leqslant x^{(0)}
$$

To obtain $\stackrel{+}{\boldsymbol{x}}$, consider solving equation $\boldsymbol{x}=\mathbf{f}^{+} \circ \mathbf{h}^{+}(\boldsymbol{x})$ by fixed-point iterations. Let $\stackrel{+}{x}^{(0)}$ be the initial point, with $\dot{x}^{(0)}=x^{(k)}$, and define the generic iteration by $\stackrel{+}{\boldsymbol{x}}^{(t)}=$ $\mathbf{f}^{+} \circ \mathbf{h}^{+}\left(\stackrel{+}{\boldsymbol{x}}^{(\mathbf{t}-1)}\right)$ for $\mathbf{t} \geqslant 1$. For cell $v$, we have $\stackrel{+}{x}_{v}^{(1)}=\mathbf{f}_{v}^{+} \circ$ $\mathbf{h}^{+}\left(\stackrel{\boldsymbol{x}}{(0)}^{(0)}=\mathrm{f}_{v}^{+} \circ \mathbf{h}^{+}\left(\boldsymbol{x}^{(\mathrm{k})}\right)\right.$. Note that $\mathrm{f}_{v}^{+} \circ \mathbf{h}^{+}\left(\boldsymbol{x}^{(\mathrm{k})}\right) \leqslant \boldsymbol{x}_{v}^{(\mathrm{k})}$. By the construction $\stackrel{+}{\boldsymbol{x}}^{(0)}=\boldsymbol{x}^{(\mathrm{k})}$ we have $\mathrm{f}_{v}^{+} \circ \mathbf{h}^{+}\left(\boldsymbol{x}^{(\mathrm{k})}\right) \leqslant$ $x_{v}^{(k)}=\stackrel{+}{x}_{v}^{(0)}$. Therefore we obtain

$$
\stackrel{+}{x}_{v}^{(1)} \leqslant \dot{x}_{v}^{(0)}
$$

For any cell $i \neq v$, recall that $f_{i}^{+} \circ \mathbf{h}^{+}(\boldsymbol{x})=\mathbf{f}_{\boldsymbol{i}} \circ \mathbf{h}^{+}(\boldsymbol{x})$ for any $\boldsymbol{x} \in \mathbb{R}_{+}^{n}$. Thus $\stackrel{+}{x}_{i}^{(1)}=\mathbf{f}_{i}^{+} \circ \mathbf{h}^{+}\left(\stackrel{+}{\boldsymbol{x}}^{(0)}\right)=\mathbf{f}_{i} \circ \mathbf{h}^{+}\left(\stackrel{+}{\boldsymbol{x}}^{(0)}\right)=$ $f_{i} \circ h^{+}\left(\boldsymbol{x}^{(k)}\right)=x_{i}^{(k+1)}$. Combined with Eq. (20), we have

$$
\stackrel{+}{x}_{i}^{(1)} \leqslant x_{i}^{(0)} \quad i \neq v
$$

Eq. (21) and Eq. (22) lead to the conclusion that $\dot{x}^{(1)} \leqslant \stackrel{+}{x}^{(0)}$. By Property 1, at convergence the following is true.

$$
\stackrel{+}{\boldsymbol{x}} \leqslant \cdots \leqslant \dot{\boldsymbol{x}}^{(2)} \leqslant \stackrel{+}{\boldsymbol{x}}^{(1)} \leqslant \stackrel{+}{\boldsymbol{x}}^{(0)}=\boldsymbol{x}^{(\mathrm{k})}
$$

Combined with Eq. (20), we obtain $\stackrel{+}{x} \leqslant \tilde{x}$. Hence the conclusion.

Theorem 7 provides a partial optimality condition for an optimization algorithm to examine whether or not adding a link improves the performance. Let $\chi^{(0)}$ be the load vector before adding the link between cell $v$ and UE $u$, and consider the sequence $\boldsymbol{x}^{(0)}, \boldsymbol{x}^{(1)}, \ldots$, with $\boldsymbol{x}^{(\mathrm{t})}=\mathbf{f} \circ \mathbf{h}^{+}\left(\boldsymbol{x}^{(\mathrm{t}-1)}\right)$. If the condition in the theorem holds, the load of all cells could be reduced by adding the link, yielding better objective value for both MinSumL and MinMaxL.

Theorem 8. Consider the sequence $\gamma^{(0)}, \gamma^{(1)}, \ldots$, where $\gamma^{(0)}=\tilde{\gamma}$, and $\boldsymbol{\gamma}^{(\mathrm{t})}=\mathbf{h} \circ \mathbf{f}^{-}\left(\boldsymbol{\gamma}^{(\mathrm{t}-1)}\right), \mathrm{t} \geqslant 1$. Then $\overline{\boldsymbol{x}} \leqslant \tilde{\boldsymbol{x}}$, if for any iteration $\mathrm{k} \geqslant 1$ we have $\mathrm{h}_{\bar{u}}^{-} \circ \mathbf{f}^{-}\left(\boldsymbol{\gamma}^{(\mathrm{k})}\right) \geqslant \gamma_{\mathrm{u}}^{(\mathrm{k})}$.

Proof. Suppose $h_{u}^{-} \circ f^{-}\left(\gamma^{(k)}\right) \geqslant \gamma_{u}^{(k)}$ for iteration k. By Lemma 6, we have $\boldsymbol{\gamma}^{(1)}=\mathbf{h} \circ \mathbf{f}^{-}\left(\boldsymbol{\gamma}^{(0)}\right) \geqslant \mathbf{h} \circ \mathbf{f}\left(\boldsymbol{\gamma}^{(0)}\right)$. Since $\boldsymbol{\gamma}^{(0)}=\tilde{\gamma}, \boldsymbol{\gamma}^{(0)}=\mathbf{h} \circ \mathbf{f}\left(\boldsymbol{\gamma}^{(0)}\right)$ holds. Hence $\boldsymbol{\gamma}^{(1)} \geqslant \boldsymbol{\gamma}^{(0)}$. Therefore, by Property 1 ,

$$
\gamma^{(k+1)} \geqslant \gamma^{(k)} \geqslant \gamma^{(k-1)} \geqslant \cdots \geqslant \gamma^{(1)} \geqslant \gamma^{(0)} .
$$

To obtain $\bar{\gamma}$, consider solving $\gamma=\mathbf{h}^{-} \circ \mathbf{f}^{-}(\boldsymbol{\gamma})$ by fixedpoint iterations. Let $\bar{\gamma}^{(0)}$ be the initial point, with $\bar{\gamma}^{(0)}=\gamma^{(\mathrm{k})}$, and define the generic iteration by $\bar{\gamma}^{(\mathrm{t})}=\mathbf{h}^{-} \circ \mathbf{f}^{-}\left(\overline{\boldsymbol{\gamma}}^{(\mathrm{t}-1)}\right)$, for $t \geqslant 1$. For UE $u$, we have $\bar{\gamma}_{u}^{(1)}=h_{u}^{-} \circ f^{-}\left(\bar{\gamma}^{(0)}\right)=$ $h_{\bar{u}}^{-} \circ f^{-}\left(\gamma^{(k)}\right)$. Note that $h_{\bar{u}}^{-} \circ f^{-}\left(\gamma^{(k)}\right) \leqslant \gamma_{u}^{(k+1)}$. Combined with the construction $\bar{\gamma}^{(0)}=\gamma^{(k)}$, we have $h_{u}^{-} \circ f^{-}\left(\gamma^{(k)}\right) \leqslant$ $\gamma_{u}^{(k)}=\bar{\gamma}_{u}^{(0)}$. Therefore

$$
\bar{\gamma}_{u}^{(1)} \geqslant \bar{\gamma}_{u}^{(0)}
$$

For any UE $j \neq u$, recall that $h_{j}^{-} \circ f^{-}(\gamma)=h_{j} \circ f^{-}(\gamma)$ for any $\gamma \in \mathbb{R}_{+}^{m}$. Thus $\bar{\gamma}_{j}^{(1)}=h_{j}^{-} \circ f^{-}\left(\bar{\gamma}^{(0)}\right)=h_{j} \circ f^{-}\left(\bar{\gamma}^{(0)}\right)=$ $h_{j} \circ f^{-}\left(\gamma^{(k)}\right)=\gamma_{j}^{(k+1)}$. Combined with Eq. (24), we have

$$
\bar{\gamma}_{j}^{(1)} \geqslant \gamma_{j}^{(0)} \quad j \neq u
$$

By Eq. (25) and Eq. (26), one concludes that $\bar{\gamma}^{(1)} \geqslant \bar{\gamma}^{(0)}$. By Property 1, at convergence the following holds.

$$
\bar{\gamma} \geqslant \cdots \geqslant \bar{\gamma}^{(2)} \geqslant \bar{\gamma}^{(1)} \geqslant \bar{\gamma}^{(0)}=\gamma^{(k)}
$$


Combined with Eq. (24), we obtain

$$
\bar{\gamma} \geqslant \tilde{\gamma}
$$

Utilizing the above inequality and Lemma 1, we have

$$
\mathbf{f}(\bar{\gamma}) \geqslant \mathbf{f}(\tilde{\gamma})
$$

By Lemma $3, \bar{x}=\mathbf{f}(\bar{\gamma})$ and $\tilde{\boldsymbol{x}}=\mathbf{f}(\tilde{\gamma})$. These, together with Eq. (29), give the conclusion.

Similar to Theorem 7, Theorem 8 can be used algorithmically, to examine if an improvement can be obtained from removing a link between a cell-UE pair.

The following corollaries provide two necessary conditions of load reduction.

Corollary 9. For sequence $\gamma^{(0)}, \gamma^{(1)}, \ldots$, where $\gamma^{(0)}=\tilde{\gamma}$, and $\boldsymbol{\gamma}^{(\mathrm{t})}=\mathbf{h} \circ \mathbf{f}^{+}\left(\boldsymbol{\gamma}^{(\mathrm{t}-1)}\right), \mathrm{t} \geqslant 1, \stackrel{+}{\boldsymbol{\chi}} \leqslant \tilde{\mathbf{x}}$ with strict inequality for at least one element, only if $\forall \mathrm{t} \geqslant 1, \mathrm{~h}_{\mathrm{u}}^{+} \circ \mathrm{f}^{+}\left(\gamma^{(\mathrm{t})}\right)>\gamma_{\mathrm{u}}^{(\mathrm{t})}$.

Proof. Suppose there is $k \geqslant 1$ such that $h_{u}^{+} \circ f^{+}\left(\gamma^{(k)}\right) \leqslant \gamma_{u}^{(k)}$. Following the flow of arguments in the proof of Theorem 8, $\stackrel{+}{\boldsymbol{\chi}} \geqslant \tilde{\boldsymbol{x}}$. Hence the conclusion.

Corollary 10. For sequence $\boldsymbol{x}^{(0)}, \boldsymbol{x}^{(1)}, \ldots$, where $\boldsymbol{x}^{(0)}=\tilde{\boldsymbol{x}}$, and $\boldsymbol{x}^{(\mathrm{t})}=\mathbf{f} \circ \mathbf{h}^{-}\left(\boldsymbol{x}^{(\mathrm{t}-1)}\right), \mathrm{t} \geqslant 1, \overline{\boldsymbol{x}} \leqslant \tilde{\boldsymbol{x}}$ with strict inequality for at least one element, only if $\forall \mathrm{t} \geqslant 1, \mathrm{f}_{v}^{-} \circ \mathbf{h}^{-}\left(\boldsymbol{x}^{(\mathrm{t})}\right)<\mathrm{x}_{v}^{(\mathrm{t})}$.

Proof. The proof is easily obtained by the proof of Theorem 7 and contradiction.

From an algorithmic standpoint, Corollary 9 and Corollary 10 complement Theorem 7 and Theorem 8. Namely, as the two corollaries provide necessary conditions, they can be used in an algorithm in order to conclude link adjustments that will not improve the performance.

The derived conditions do not necessarily require to account for all cells and UEs. This fact potentially enables to examine the conditions to a cell and its local environment. Suppose we add a link for cell $v$ and UE $u$. For any subset $\breve{\mathcal{J}} \subset \mathcal{J}$ with $v \in \breve{\mathcal{J}}$, and the relevant set of UEs $\breve{J}=\bigcup_{i \in \breve{J}} \mathcal{J}_{i}$, consider the sufficient condition in Theorem 7 . We argue that the condition can be used, even if we fix the load of all cells in $\mathcal{J} \backslash \breve{J}$ and perform the so called asynchronous fixed-point iteration [26] to function $\mathbf{f} \circ \mathbf{h}^{+}(\boldsymbol{x})$ for the load of cells in $\breve{\mathcal{J}}$, as formalized below.

Theorem 11. Consider the sequence $\breve{\boldsymbol{x}}^{(0)}, \breve{x}^{(1)}, \ldots$, generated by the following asynchronous fixed-point iterations.

1) $\breve{x}^{(0)}=\tilde{x}$.

2) For any $i \in \mathcal{J} \backslash \breve{\mathcal{J}}$ and $\mathrm{t} \geqslant 1, \breve{x}_{i}^{(t)}=\breve{x}_{i}^{(0)}$.

3) For any $i \in \breve{\mathcal{J}}$ and $\mathrm{t} \geqslant 1, \breve{x}_{i}^{(t)}=f_{i} \circ \mathbf{h}^{+}\left(\breve{x}^{(t-1)}\right)$.

Then $\stackrel{+}{x} \leqslant \tilde{x}$ if for some iteration $\mathrm{k} \geqslant 1$ we have $\mathrm{f}_{v}^{+}$。 $\mathbf{h}^{+}\left(\breve{\boldsymbol{x}}^{(\mathrm{k})}\right)<\breve{\mathbf{x}}_{v}^{(\mathrm{k})}$.

Proof. Suppose there is an iterate $\breve{x}^{(k)}$, for some $k \geqslant 1$ such that $\mathrm{f}_{v}^{+} \circ \mathbf{h}^{+}\left(\breve{\boldsymbol{x}}^{(\mathrm{k})}\right)<\breve{\boldsymbol{x}}_{v}^{(\mathrm{k})}$. By Lemma 5, we have $f_{i} \circ \mathbf{h}^{+}\left(\breve{\boldsymbol{x}}^{(0)}\right) \leqslant \mathrm{f}_{\mathrm{i}} \circ \mathbf{h}\left(\breve{\boldsymbol{x}}^{(0)}\right)$, which leads to $\breve{x}_{i}^{(1)} \leqslant \breve{x}_{\dot{i}}^{(0)}$, for any $i \in \breve{\mathcal{J}}$. Combined with condition 2) that $\breve{x}_{i}^{(1)}=\breve{x}_{i}^{(0)}$ for any $i \in \mathcal{J} \backslash \breve{\mathcal{J}}$, we conclude that $\breve{\boldsymbol{x}}^{(1)} \leqslant \breve{\boldsymbol{x}}^{(0)}$. For any $i \in \breve{\mathcal{J}}$, by applying Property 1 on $\breve{x}_{i}^{(2)}$, we obtain $\breve{x}_{i}^{(2)} \leqslant \breve{x}_{i}^{(1)}$. Again, combine the result with condition 2 ), and repeat the procedure for the remaining iterations, one can conclude

$$
\breve{\boldsymbol{x}}^{(k+1)} \leqslant \breve{\boldsymbol{x}}^{(k)} \leqslant \breve{\boldsymbol{x}}^{(k-1)} \leqslant \cdots \leqslant \breve{\boldsymbol{x}}^{(1)} \leqslant \breve{\boldsymbol{x}}_{\mathfrak{i}}^{(0)}
$$

Suppose we apply regular (instead of asynchronous) fixedpoint iterations to $\mathbf{f}^{+} \circ \mathbf{h}^{+}(\boldsymbol{x})$, using $\breve{\boldsymbol{x}}^{(\mathrm{k})}$ as the initial point. This gives a sequence $\stackrel{+}{\boldsymbol{x}}^{(0)}, \stackrel{+}{\boldsymbol{x}}^{(1)}, \ldots$, with $\stackrel{+}{\boldsymbol{x}}^{(0)}=\breve{\boldsymbol{x}}^{(k)}$. For cell $v, \dot{x}_{v}^{(1)}<\stackrel{+}{x}(0)_{v}=\breve{x}_{v}^{(k)}$, because $f_{v}^{+} \circ \mathbf{h}^{+}\left(\breve{\mathbf{x}}^{(k)}\right)<\breve{x}_{v}^{(k)}$ and $\stackrel{+}{x}_{v}^{(1)}=f_{v}^{+} \circ h^{+}\left(\breve{x}^{(k)}\right)$. For any cell $i \neq v$, note that $f_{i}^{+} \circ h^{+}(x)=f_{i} \circ h^{+}(x), x \in \mathbb{R}_{+}^{n}$. Hence $f_{i}^{+} \circ h^{+}\left(x^{(0)}\right)=f_{i} \circ$ $\mathbf{h}^{+}\left(\stackrel{+}{\boldsymbol{x}}^{(0)}\right)$. Moreover, $\boldsymbol{f}_{i}^{+} \circ \mathbf{h}^{+}\left(\stackrel{+}{\boldsymbol{x}}^{(0)}\right)=\dot{\boldsymbol{x}}_{i}^{(1)}$, and $\boldsymbol{f}_{\boldsymbol{i}} \circ \mathbf{h}^{+}\left(\dot{\boldsymbol{x}}^{(0)}\right)=$ $\breve{x}_{i}^{(k)}$. Thus $\stackrel{+}{x}_{i}^{(1)}=\breve{x}_{i}^{(k)}$, and by construction $\stackrel{+}{x}_{i}^{(0)}=\breve{x}_{i}^{(k)}$. In conclusion, $\dot{x}^{(1)} \leqslant \dot{x}^{(0)}=\breve{\boldsymbol{x}}^{(\mathrm{k})}$. The conclusion and Property 1 lead to $\stackrel{+}{x} \leqslant \tilde{x}$.

\section{Algorithm Design}

In Algorithm 2 and Algorithm 3, we apply the conditions in Section V-B to examine possible load reduction by one link adjustment. Since the steps of two algorithms are similar to each other, we omit some details in the description of Algorithm 3.

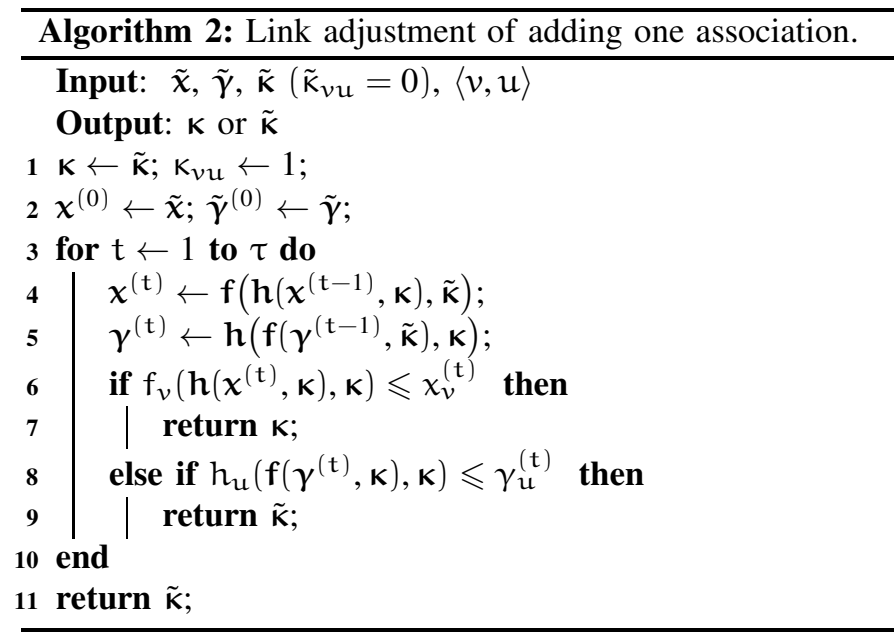

The input of Algorithm 2 consists of an association $\tilde{\mathrm{K}}$, the load $\tilde{\chi}$ and the SINR $\tilde{\gamma}$ for association $\tilde{\kappa}$, and a cell-UE pair $\langle v, u\rangle$, for which $\tilde{\mathrm{K}}_{v u}=0$ and hence it is subject to the consideration of adding the association. Parameter $\tau$ is pre-defined, and denotes the maximum number of iterations of applying the conditions in Theorem 7 and Corollary 9. Lines 6 and 8 apply, respectively, the conditions provided in 


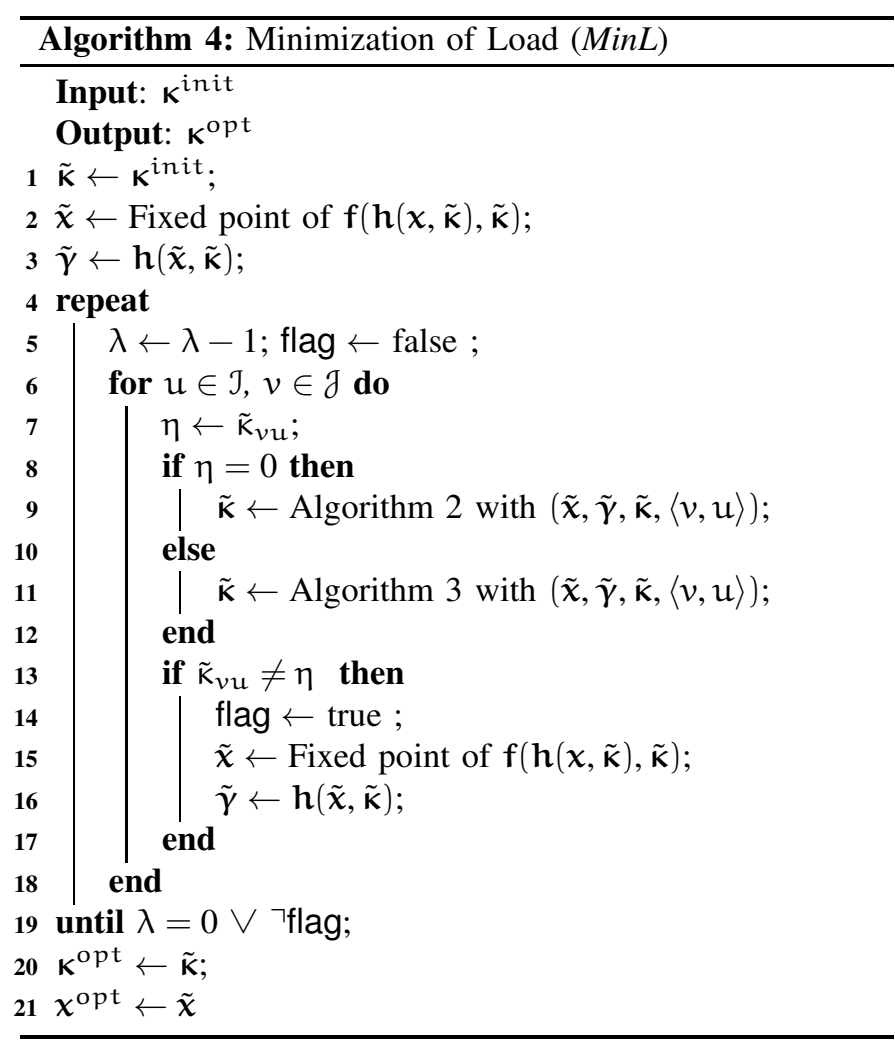

Theorem 7 and Corollary 9. If the outcome of Line 6 turns out to be true, then adding the association $\langle v, \mathfrak{u}\rangle$ improves the load of all cells by Theorem 7, and Algorithm 2 updates the cell-UE association to include this pair. If Line 8 holds true, then the necessary condition for load improvement in Corollary 9 is not met. Consequently $\langle v, u\rangle$ is rejected and the original association $\tilde{\kappa}$ with $\tilde{\kappa}_{v u}=0$ is kept. This association is also kept in case neither the sufficient nor the necessary conditions is met. Note that in considering the removal of a link, the conditions in Theorem 8 and Corollary 10 are used in Algorithm 3.

The overall algorithm, named MinL, is presented in Algorithm 4 . The outer loop runs for $\lambda$ rounds, where $\lambda$ is a predefined parameter. The inner loop goes through all the cell-UE pairs. For each pair, Algorithm 2 or 3 is utilized to evaluate the corresponding link adjustment. The indicator flag, is initialized to be false, at the beginning of each outer loop round. The outcome of Algorithm 2 and 3 is examined in Line 13. If there is an improvement, the load and SINR of the updated association $\tilde{\kappa}$ are computed, and flag is set to be true, before moving to the next cell-UE pair. For each outer loop round, if there is no improvement throughout all its inner loop rounds, then flag is not updated and kept false, which, causes the outer loop to terminate.

As for algorithm complexity, we observe first that the complexity of Algorithm 2 and Algorithm 3, in terms of evaluating fixed-point solutions, is clearly of $\mathrm{O}(\tau \mathrm{m}+\tau \mathrm{n})$. Recall that $m$ and $n$ are the numbers of users and cells, respectively. In Algorithm 4, Algorithm 2 (or Algorithm 3) is invoked $\lambda \mathrm{mn}$ times at maximum. Also, the fixed point evaluations in Algorithm 4 in Line 15 are executed $\lambda \mathrm{mn}$ times at most.
The complexity of the loop in Algorithm 4 is thus $\mathrm{O}(\lambda \mathrm{mn}) \times$ $\mathrm{O}(\tau \mathrm{m}+\tau n+\mathrm{Kn})=\mathrm{O}\left(\lambda \tau \mathrm{m}^{2} \mathrm{n}+\lambda \tau m n^{2}+\lambda m n^{2} \mathrm{~K}\right)$, where $\mathrm{K}$ is the number of fixed-point iterations per evaluation. For standard interference functions, the fixed-point method has been shown to have geometric convergence and does not generate the issue of computational bottleneck (see [27]). Moreover, out of the loop of Algorithm 4, in Line 2, we obtain the fixed point of the load-coupling function under the initial association. Therefore, the overall complexity of Algorithm 4 is $\mathrm{O}\left(\mathrm{Kn}+\lambda \tau \mathrm{m}^{2} \mathrm{n}+\lambda \tau m n^{2}+\lambda m \mathrm{n}^{2} \mathrm{~K}\right)=\mathrm{O}\left(\lambda \tau \mathrm{m}^{2} n+\lambda \tau m n^{2}+\right.$ $\lambda m n^{2} \mathrm{~K}$ ), which is polynomial in the input size, the number of fixed-point solutions, and algorithm control parameters. Hence the algorithm is scalable.

We remark that MinL, presented in Algorithm 4, suits well for distributed implementation. By Theorem 11, by means of asynchronous fixed-point iterations, the results of Theorem 7, Theorem 8, Corollary 9, and Corollary 10, apply also to any subset of cells. Therefore, for Algorithm 4, a distributed implementation amounts to, for any specific association of cell $v$ and UE $u$, examining the partial optimality conditions for a cell subset $\breve{\mathcal{J}}$ with $v \in \breve{\mathcal{J}}$ (cf. Theorem 8). Such an implementation is hence straightforward in concept. Here, subset $\breve{\mathcal{J}}$ is naturally formed by the neighboring cells of $v$, because more remote cells have virtually no significance in interference. Thus, the distributed implementation only requires information exchange that is local to a cell and hence easily managed via the X2 interface.

\section{LOWER Bounds of Global Optimum}

In this section, we show how to derive lower bounds of the global optimum, for both MinSumL and MinMaxL, based on the linearization in Section IV. Such lower bounds provide effective means to gauge of the performance of the solution approaches presented in Sections IV and V.

Consider the linear approximation derived using the two end points 0 and $T_{\ell, j}$, where $j$ and $\ell \in \mathcal{L}_{j}$ are the UE and its set of serving cells under consideration, and $T_{\ell, j}$ is defined in Eq. (12). The linear approximation is illustrated in Figure 3. Similar to Figure 2, the specific values of the axis are not shown in Figure 3 for the sake of generality. Constructing the linear approximation for all UEs and their candidate serving cells, the resulting MILP formulations $S_{-}$ $M I L P$ and $M-M I L P$ will provide lower bounds of the global optimum of MinSumL and MinMaxL, respectively. This is simply because, no matter the association, the interference is always within the interval used for constructing the linear function, and the load calculated by the linear function is an underestimation of the load for the entire interval.

The gap between the original, nonlinear load function and the linear function as constructed above can be very significant, as can be seen in Figure 3. To overcome the issue, we derive a better approximation by reducing the domain of the interference variable $w_{\ell, j}, \ell \in \mathcal{L}_{j}, j \in \mathcal{J}$, with the theoretical guarantee that the interference, if UE $j$ is served by cells $\ell$ at the global optimum, will indeed fall within the reduced domain. Consequently, the linear function derived by the reduced interference domain remains an underestimation 
of the load of the global optimum, whereas the quality of the approximation is improved. To this end, we first derive lower and upper bounds for the load vector $x$ in Lemma 12 below.

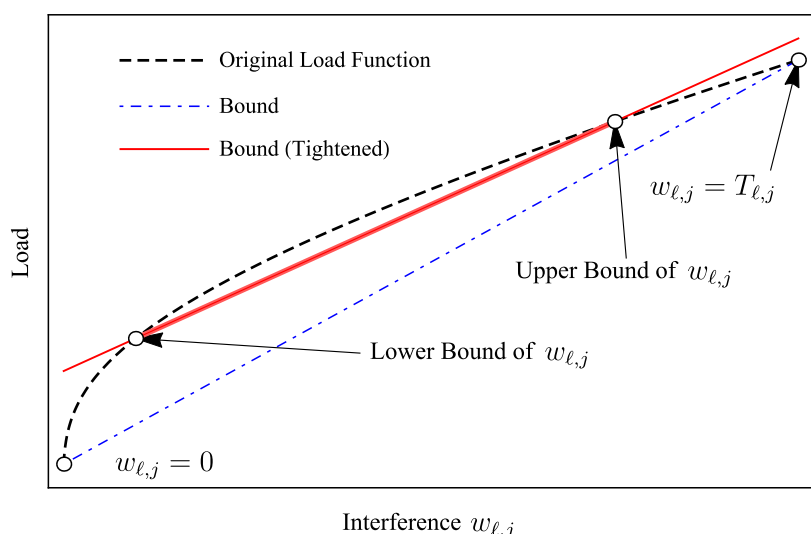

Figure 3. The lower bound can be improved by reducing the range of relevance for the interference variable.

\section{A. Bounds of Cell Load}

Lemma 12. Define two matrices $\hat{\kappa}$ and $\check{\kappa}$. For $\hat{\kappa}$, entry $\hat{\kappa}_{i j}=1$ if $i=c_{j}$, and zero otherwise. For $\check{\kappa}$, entry $\check{\mathrm{K}}_{i j}=1$ if $\mathrm{j} \in \mathrm{J}_{i}^{+}$, and zero otherwise. Denote by $\boldsymbol{x}$ and $\stackrel{\circ}{\chi}$ the fixed points of $\boldsymbol{x}=\mathbf{f}(\mathbf{h}(\boldsymbol{x}, \check{\mathbf{k}}), \hat{\mathbf{k}})$ and $\boldsymbol{x}=\mathbf{f}(\mathbf{h}(\boldsymbol{x}, \hat{\mathbf{k}}), \check{\mathbf{k}})$, respectively. For any cell-UE association, the incurred cell load vector is bounded from below and above, respectively, by $\underset{0}{x}$ and $\stackrel{\circ}{x}$.

Proof. Consider first $\chi x$. For any given load vector $x$ and an association vector $\boldsymbol{\kappa}$, it can be verified that $\mathbf{h}(\boldsymbol{x}, \check{\boldsymbol{\kappa}}) \geqslant \mathbf{h}(\boldsymbol{x}, \boldsymbol{\kappa})$. This is because, for any UE $j \in \mathcal{J}$, all the candidate cells in $\mathcal{J}_{j}^{+}$are serving $\boldsymbol{j}$ in $\check{\kappa}$. Moreover, for any $\gamma, \mathbf{f}(\gamma, \hat{\kappa}) \leqslant \mathbf{f}(\gamma, \boldsymbol{\kappa})$, because for any cell $i \in \mathcal{J}$, the load $x_{i}$ originates only from serving the UEs in $\mathcal{J}_{\boldsymbol{i}}^{-}$, for which $i$ is the home cell. Hence $\mathbf{f}(\mathbf{h}(\boldsymbol{x}, \check{\mathbf{k}}), \hat{\mathbf{k}}) \leqslant \mathbf{f}(\mathbf{h}(\boldsymbol{\chi}, \boldsymbol{\kappa}), \boldsymbol{\kappa})$ holds for any $\boldsymbol{\kappa}$ and $\boldsymbol{x}$. This establishes the validity of $x$ as lower bound of cell load. The validity of $\stackrel{\circ}{\chi}$ as upper bound can be proven analogously, by observing that $\mathbf{h}(\boldsymbol{x}, \hat{\mathbf{k}}) \leqslant \mathbf{h}(\boldsymbol{x}, \boldsymbol{\kappa})$ and $\mathbf{f}(\boldsymbol{\gamma}, \check{\boldsymbol{\kappa}}) \geqslant \mathbf{f}(\boldsymbol{\gamma}, \boldsymbol{\kappa})$, which together lead to $\mathbf{f}(\mathbf{h}(\boldsymbol{x}, \hat{\mathbf{k}}), \check{\mathbf{k}}) \geqslant \mathbf{f}(\mathbf{h}(\boldsymbol{x}, \boldsymbol{\kappa}), \mathbf{\kappa})$.

Intuitively, the lower bound $\underset{0}{x}$ given by Lemma 12 is rather loose, because it is derived by assuming the most optimistic interference conditions which are fully unrealistic. Nevertheless, the two bounds on $x$ can be utilized together to reduce the domain of interest for the interference variables, and thus improving the approximation as discussed earlier. One can refer to Figure 3 for an illustration. Below, theoretical insights are given, ensuring that the strengthened linear approximation remains valid in delivering a lower bound to the global optimum.

\section{B. Validity of the Improved Lower Bound}

Corollary 13. For any $U E j$ and $\ell \in \mathcal{L}_{j}, w_{\ell, j} \in\left[W_{\ell, j}, \stackrel{\circ}{W}_{\ell, j}\right]$, with $W_{\ell}, j=\sum_{i \in \mathcal{J} \backslash \ell} p_{i} g_{i j} \chi_{i}$ and $\stackrel{\circ}{W}_{\ell, j}=\sum_{i \in \mathcal{J} \backslash \ell} p_{i} g_{i j} \stackrel{\circ}{x}_{i}$.
Proof. Since both $p_{i}$ and $g_{i j}$ are constant for any $i \in \mathcal{J}$ and $j \in \mathcal{J}$, this result follows directly from Lemma 12 .

Theorem 14. (Lower bound) The optimum of $S-M I L P$ is a lower bound of MinSumL, with the two constants $s_{\ell, j}$ and $\mu_{\ell, j}$ as defined in in Corollary 13, and the following additional constraint

$$
w_{\ell, j} \geqslant W_{\ell, j}-T\left(1-\kappa_{\ell, j}\right) \quad \ell \in \mathcal{L}_{j}, j \in \mathcal{J}
$$

Proof. The extra constraint shown in Eq. (31) guarantees that we have $w_{\ell, j} \geqslant \max \left\{W_{\ell, j}, \sum_{i \in \mathcal{J} \backslash \ell} p_{i} g_{i j} x_{i}\right\}$ for any $\ell \in \mathcal{L}_{j}$ and $j \in \mathcal{J}$, if $\kappa_{\ell, j}=1$. That is, if $\sum_{i \in \mathcal{J} \backslash \ell} p_{i} g_{i j} x_{i}<W_{\ell, j}$, then $w_{\ell, j}$ is equal to its lower bound $W_{\ell, j}$. Otherwise, we have $w_{\ell, j}=\sum_{i \in \mathcal{J} \backslash \ell} p_{i} g_{i j} x_{i}$

Consider the optimal load solution in MinSumL. For any UE $j$, suppose its serving cells set is $\ell \in \mathcal{L}_{j}$. By construction of the linear function, the interference $w_{\ell, j}$ satisfies $l_{\ell, j}\left(w_{\ell, j}\right) \leqslant$ $f_{\ell, j}\left(w_{\ell, j}\right)$, for interval $\left[W_{\ell, j}, \stackrel{\circ}{\ell}_{\ell, j}\right]$. Therefore, for the optimal solution of MinSumL, the objective value of $S-M I L P$ is a lower bound with the same association. This association solution is not necessarily the optimum of $S-M I L P$. Because $S-M I L P$ is minimization, the theorem follows.

For MinMaxL and its approximation $M-M I L P$, the validity of the lower bound can be proved in the same way.

\section{Performance Evaluation}

\section{A. Simulation Settings}

The network scenario consists in 19 hexagonal regions (see Figure 4), each of which has one MC in the center. In each hexagon, two SCs and thirty UEs are randomly distributed. The hexagon radius is $500 \mathrm{~m}$. The HetNet operates at $2 \mathrm{GHz}$. Each resource block follows the LTE standard of $180 \mathrm{kHz}$ bandwidth and the total bandwidth is $20 \mathrm{MHz}$ [28]. The transmit power per resource block for MCs and SCs equal $400 \mathrm{~mW}$ and $50 \mathrm{~mW}$, respectively. The noise power spectral density is $-174 \mathrm{dBm} / \mathrm{Hz}$. The path loss for MCs and SCs follow the standard 3GPP urban macro (UMa) model and urban micro (UMi) model of hexagonal deployment, with the shadowing coefficients generated by the log-normal distribution with $6 \mathrm{~dB}$ and $3 \mathrm{~dB}$ standard deviation, respectively [29]. For any UE, we set 3 candidate cells (MCs and/or SCs) and thus $\left|\mathcal{J}_{j}^{+}\right|=3, j \in \mathcal{J}$. In the MILP-based solutions, the linearization follows Corollary 13. In MinL, the parameters $\lambda$ and $\tau$ are set to 3 and 5 , respectively. As a baseline solution, each $\mathrm{UE}$ is served only by the home cell (MC or SC) with the best received signal power, without any JT. MinL is evaluated with two different initial associations: 1) the baseline solution, and 2) the association solution obtained from solving MILPs. The latter is labeled as " $-M I L P+M i n L "$ and "M-MILP + MinL". The simulations run on 20 data sets, and the results are averaged over the data sets.

\section{B. An Illustration of Optimized Association}

An example network layout is shown in Figure 4. Figure 4(a) shows the initial association, and Figure 4(b) shows the association optimized by $S-M I L P+M i n L$. The MCs 


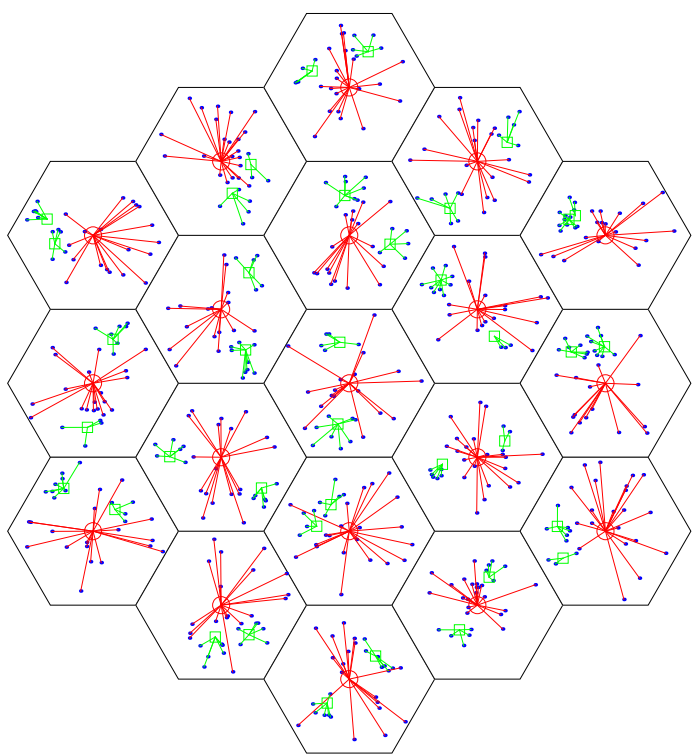

(a) Initial Association

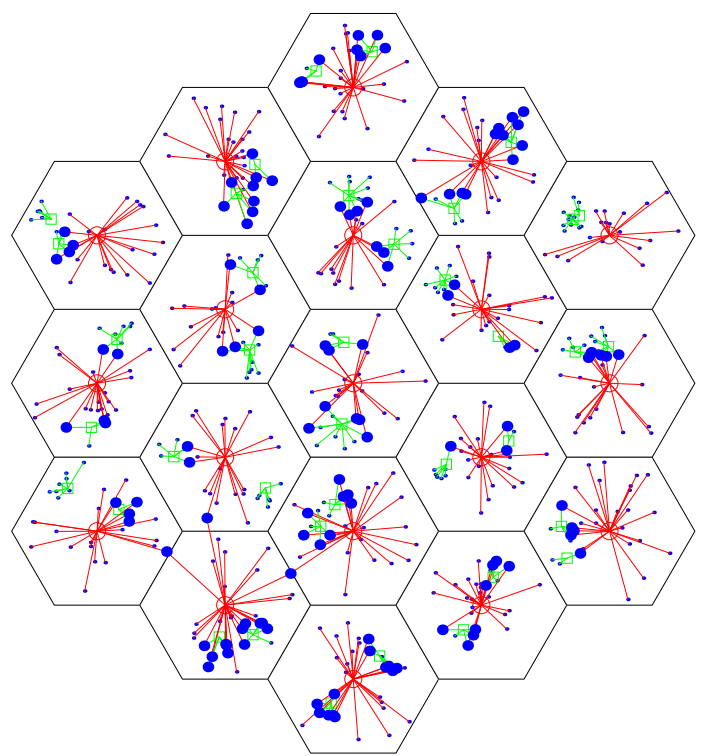

(b) Optimized Association

Figure 4. An example of the HetNet layout, and initial and the optimized association. Red circles in the center of each hexagon denote the MC. Green rectangles denote the SC. The UEs are denoted by blue dots. Figure 4(a) shows the network layout and initial association , where each UE is associated only to its home cell. Figure 4(b) shows the optimized association, where UEs served by multiple cells using JT are marked by thicker blue dots.

are marked by red circles, the SCs are marked by green rectangles, and the UEs are represented by blue dots. The red and green lines show the association of MCs and SCs to UEs, respectively. In Figure 4(b), the UEs in JT are marked by thicker dots. One can see from Figure 4 that most cooperations by JT are between MCs and SCs, whereas JT of MCs occur for a few UEs on MC edges only. From the figure, we infer that JT is likely to take place if at least two cells provide comparable received powers to a UE. In addition, it can be seen that the number of UEs in JT varies much over the area, reflecting the heterogeneity of the scenario. Also, although not shown in the figure, the simulations indicate that the number of UEs in JT increases with respect to the demand.

\section{Performance Comparison for MinSumL}

In Figure 5, we show the performance of the algorithms for MinSumL. The main results of MinSumL are given in Figure 5(a). For low UE demand, all cells are lightly loaded in the baseline solution of Non-JT, and, from the lower bound, one concludes that the amount of possible improvement is negligible. The impact of optimization becomes much apparent and very significant with high user demand. For the highest user demand in the figure, $\operatorname{MinL}$ reduces the sum load by $10 \%$, compared to the baseline, and the improvements by using $S$ $M I L P$ and $S-M I L P+M i n L$, are $15 \%$ and $17.5 \%$, respectively. Note that even for global optimum, the improvement can be no more than $18 \%$, as shown by the lower bound, which is on the optimistic side of the global optimum. Thus, from MinL, $S-M I L P$, to $S-M I L P+M i n L$, the performance progressively improve, and the latter is near optimal. We remark that the observation applies also if the results are averaged over the demand range.
To gain further insights of the results in Figure 5(a), we show the sum load of MCs and SCs separately in Figure 5(b) and (c), respectively. These two figures reveal that, in comparison to the baseline solution, the load of SCs due to optimization is significantly reduced, whereas the optimized MC load increases slightly. One can infer that, in order to minimize the sum load, MCs expand, by using JT to serve some UEs of which the home cells are SCs. This is because the load of MC and SC are equally important in the objective function in question, but the number of SCs are twice of MCs. Thus, in optimizing the sum load, the MCs tend to have higher load than the baseline solution. This insight explains why the $S-M I L P$ performs better than MinL in Figure 5(a), as the latter is designed based on the partial optimality conditions for monotonically reducing cell load. On the other hand, $\operatorname{MinL}$ is able to complement $S-M I L P$, as demonstrated by the fact that the combined use of the two achieves the best performance.

\section{Performance Comparison for MinMaxL}

The performance of the algorithms for MinMaxL is shown in Figure 6. The results are structured in three subfigures, following the construction of Figure 5. From Figure 6(a), for MinMaxL the performance gain by the proposed algorithms is considerably higher than MinSumL. For the highest user demand under consideration, MinL reduces the maximum load by almost $23 \%$, compared to the baseline, and the improvements via $M-M I L P$ and $M-M I L P+M i n L$ grow to $34 \%$ and $37 \%$, respectively. Moreover, similar to Figure 5(a), M-MILP $+\operatorname{Min} L$ performs closely to the lower bound, reinforcing the observation of its near optimality. That is, there is little room left for further performance improvement.

We argue that MCs benefit considerably more than SCs from JT in MinMaxL. By inspecting Figure 6(b) and (c), it 


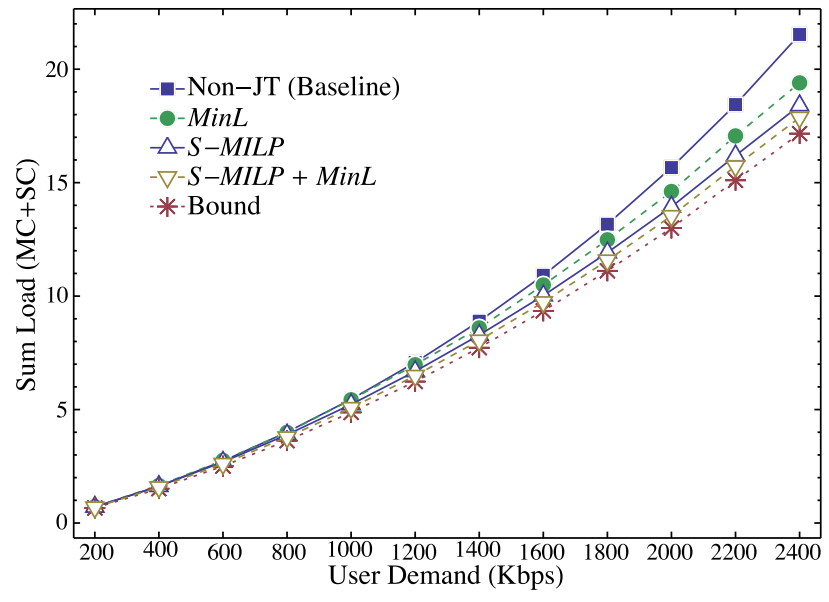

(a) Sum Load (MC+SC)

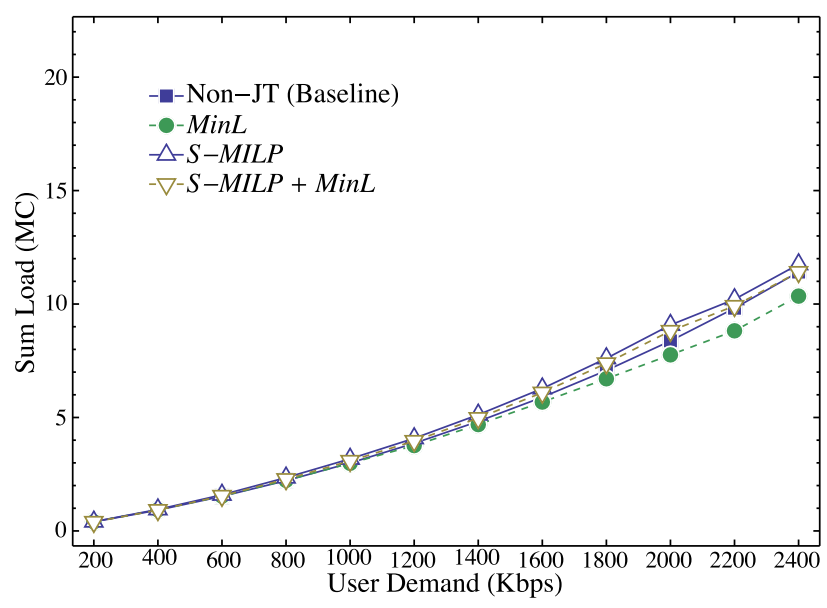

(b) Sum Load (MC)

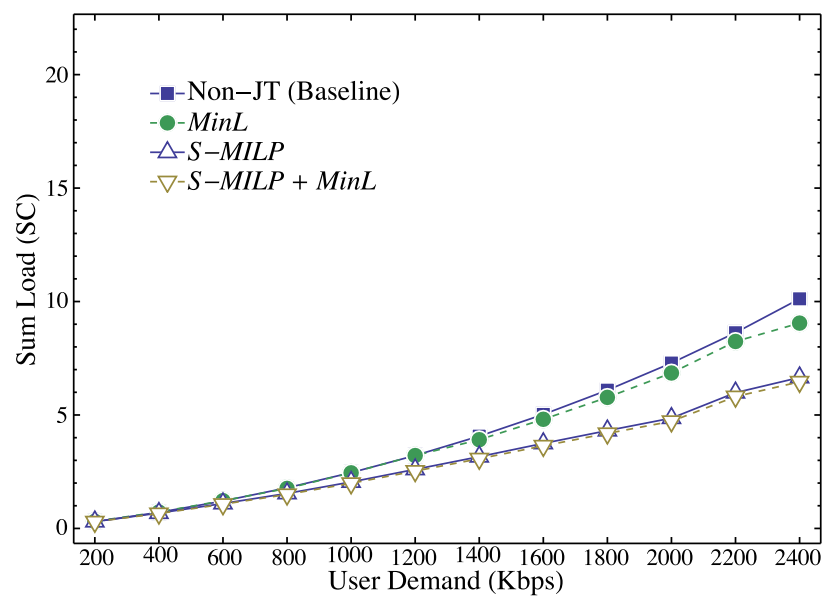

(c) Sum Load (SC)

Figure 5. Performance of sum load vs. user demand.

is apparent that algorithm $\operatorname{Min} L$ reduces the maximum load mainly for the MCs. For the SCs, the reduction of maximum load by algorithm MinL is almost negligible. From the results

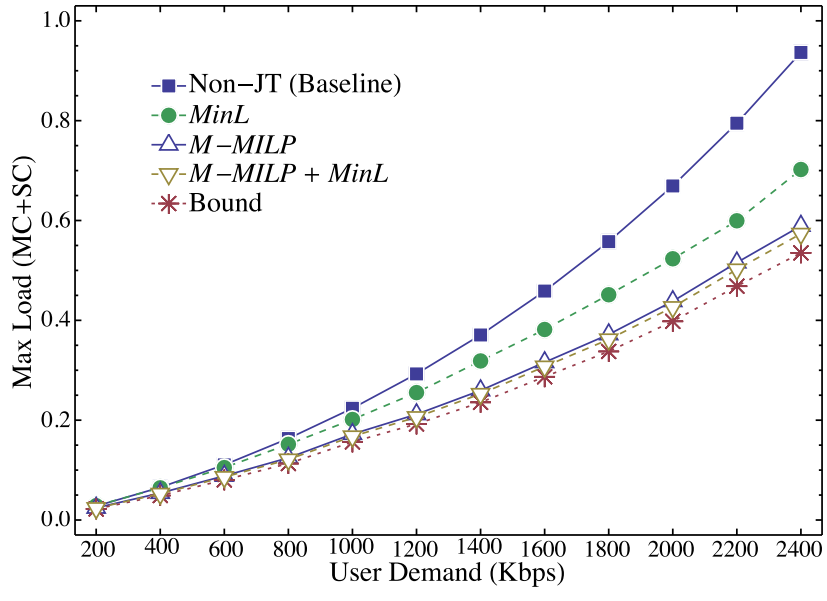

(a) Max Load (MC+SC)

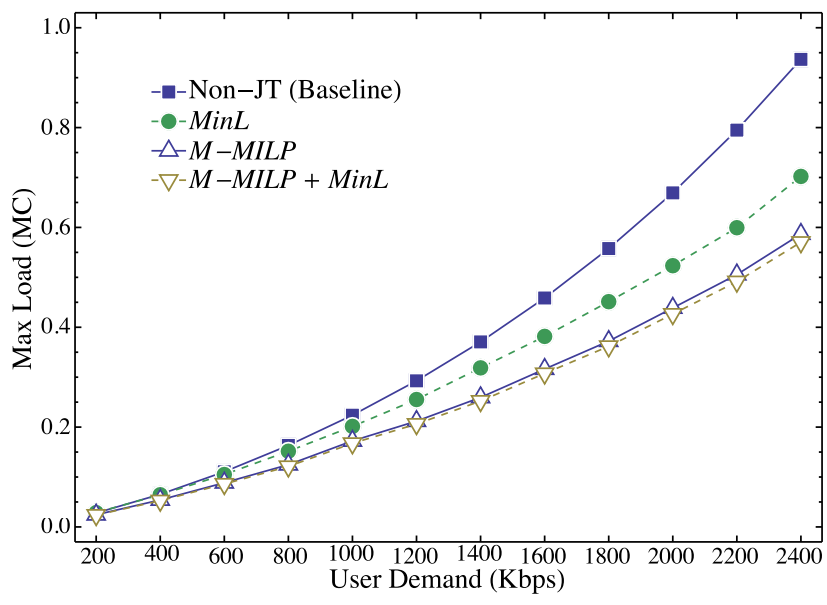

(b) Max Load (MC)

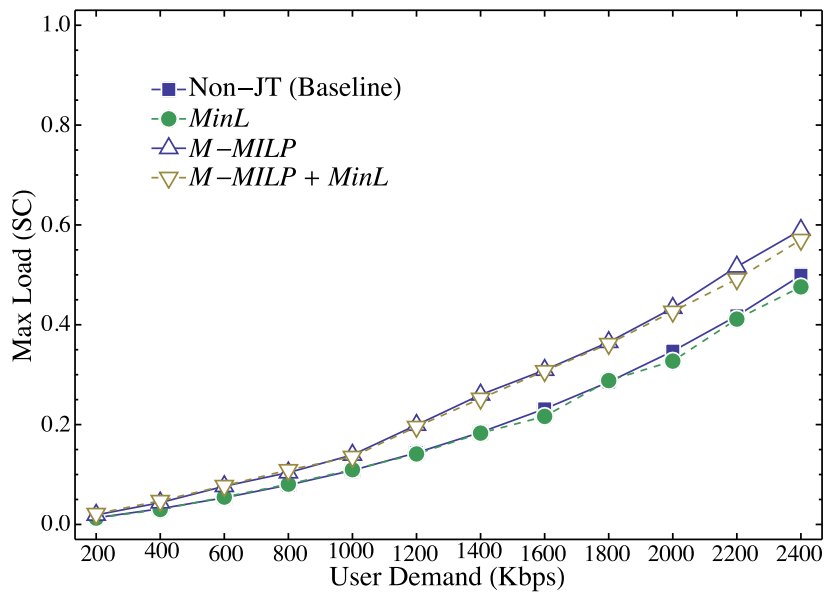

(c) Max Load (SC)

Figure 6. Performance of max load vs. user demand.

of the two MILP-based solutions, one discovers that the overall maximum load should be improved at the cost of allowing higher SC load than the baseline. In HetNets, MCs typically 
stand for higher load than SCs by the default association strategy based on received power (i.e., our baseline solution). Thus reducing the maximum load has to rely on the offloading effect of SCs, and this effect is achieved via participation of SCs in JT.

The results in Figure 6 also illustrate the trade-off between optimality and complexity. Using the low-complexity MinL (Algorithm 4), the performance improvement amounts to $23 \%$, whereas the improvement grows to $34 \%$ with M-MILP (Algorithm 1), with the price that the latter has high complexity due to the use of MILP.

In general, the true optimality gaps of $S-M I L P$ and $M-M I L P$ (or any sub-optimal algorithm) are difficult to obtain, because doing so requires the value of the global optimum that is not known. However, by selecting the end points as detailed in Section VI, $S-M I L P$ and $M-M I L P$ are also effective bounding schemes for performance evaluation, because the gap to the optimum cannot exceed the gap in relation to the bound. From the empirical results in Figure 5(a) and Figure 6(a), S-MILP and $M-M I L P$ perform well in delivering a close-to-optimal solution, because the average gap to the bounds is small, with an average of merely $3 \%$.

Recall that an alternative linear approximation for both $S$ $M I L P$ and $M-M I L P$ is to use the first-order Taylor series. We have performed additional simulations with this approximation, in which the derivative is taken at the middle point of the interference interval considered. The results show that this linear approximation has almost identical performance as the linear approximation discussed in Section IV.

\section{E. Algorithm Scalability}

In Figure 7, we show additional results for larger-scale scenarios via densification. Specifically, both the numbers of UEs and SCs are doubled. For these scenarios, we compare our scalable algorithm MinL with the baseline solution of Non-JT. For sum load, we consider normalized values with respect to the number of cells, i.e., the average load, because the number of cells in the figure differs by curve. Overall, the results for both MinSumL and MinMaxL show the same trends as those exhibited in Sections VII-C and VII-D. There are however some additional insights. For average load, the optimization algorithm yields obvious improvements. When the number of SCs is doubled, however, the improvement is insignificant. This is because, with a dense network deployment, a UE is very likely to have very good link to its home cell, and hence JT does not exhibit much benefit in average. Interestingly, for reducing maximum load, the results are quite the opposite: The improvement due to optimization is much more significant for dense cell deployment. The reason is that additional cells increase the dimension of freedom in optimizing JT to offload the most loaded cell, whereas for a sparse network, each cell acts as the home cell of quite many UEs for which there are no other cell having comparable link quality and hence there is little room for offloading via JT. Therefore, our optimization algorithm is useful for both sparse and dense scenarios for minimizing the average and maximum cell load, respectively.

In Section II, we commented on the trade-off between accuracy and complexity in interference modeling. In order

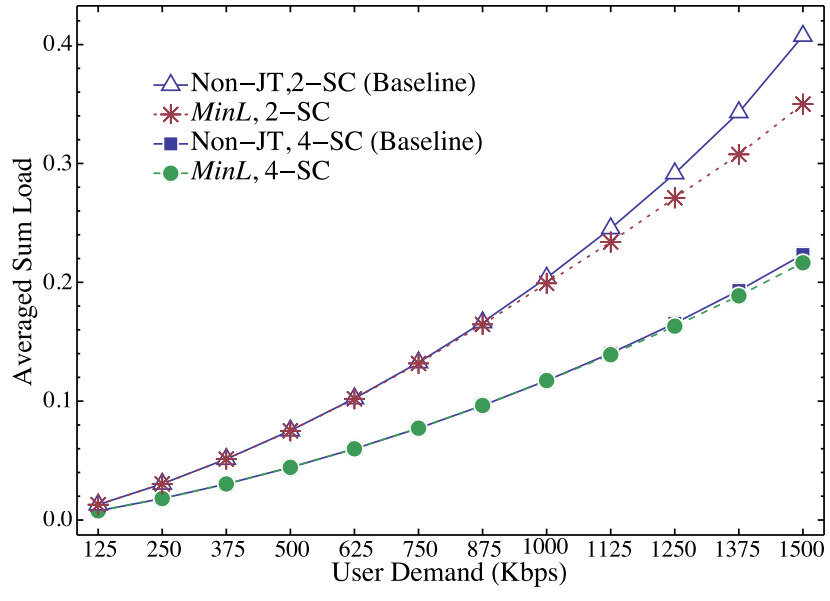

(a) Averaged Sum Load

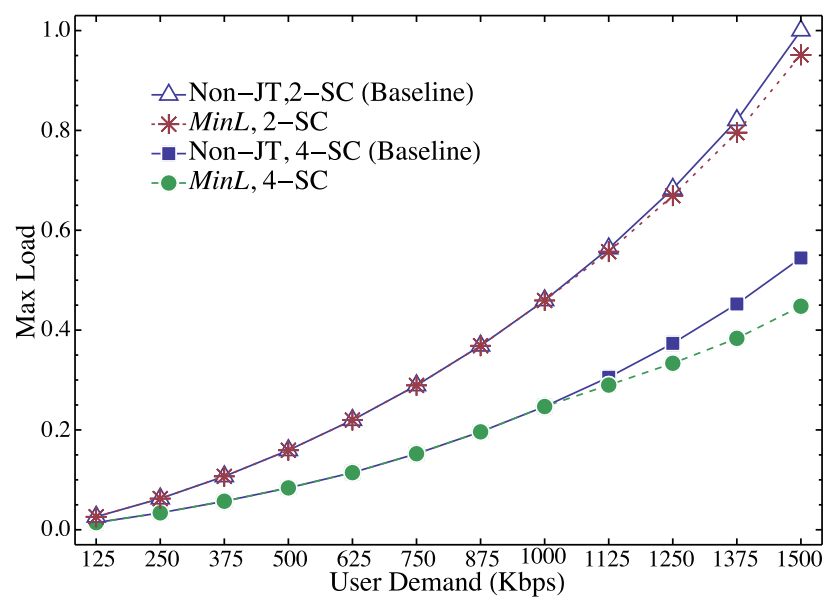

(b) Maximum Load

Figure 7. Numerical results with large network scale (60 UEs, 2 or 4 SCs in each hexagonal region).

to examine the accuracy in the modeling approach, we have performed post-processing to the solutions obtained for all the network scenarios and instances, using the more exact interference expression. The results from post-processing exhibits an average deviation of $0.7 \%$ in the performance values (cell load levels). Hence the modeling approach used is indeed reasonable.

\section{ACKNOWLEDGEMENT}

This work has been supported by the Swedish Research Council and the Linköping-Lund Excellence Center in Information Technology (ELLIIT), Sweden, and the European Union Marie Curie project MESH-WISE (FP7-PEOPLE2012-IAPP: 324515), DECADE (H2020-MSCA-2014-RISE: 645705), and WINDOW (FP7-MSCA-2012-RISE: 318992). The work of D. Yuan has been carried out within European FP7 Marie Curie IOF project 329313. 


\section{CONCLUSION}

The paper has investigated how to optimize cell-UE association with JT for load-coupled LTE HetNets. The cell load refers to the amount of time-frequency resource for serving user demand, and the cells influence each others' load levels due to mutual interference. Two optimization problems, MinSumL and MinMaxL, have been formulated and analyzed. Solutions to the two problems strive for the overall resource efficiency and load balancing among cells, respectively. We have thoroughly proved the $\mathcal{N} \mathcal{P}$-hardness of the two problems. A linearization scheme has been developed, which yields an MILP-based solution approach to the two problems. The approach also enables to compute lower bounds of global optimum. Moreover, several optimality conditions have been derived, leading to a second solution approach. Simulation results show the benefits of JT for improving resource efficiency and load balancing, and the improvement is significant for high user demand. The results also demonstrate that a combined use of the two approaches performs closely to global optimum.

\section{REFERENCES}

[1] J. G. Andrews, S. Buzzi, W. Choi, S. V. Hanly, A. Lozano, A. C. K. Soong, and J. C. Zhang, "What will 5G be?" IEEE Journal on Selected Areas in Communications, 32(6): 1065-1082, 2014.

[2] V. Jungnickel, K. Manolakis, W. Zirwas, B. Panzner, V. Braun, M. Lossow, M. Sternad, R. Apelfröjd, and T. Svensson, "The role of small cells, coordinated multipoint, and massive MIMO in 5G", IEEE Communications Magazine, 52(5): 44-51, 2014.

[3] I. Siomina and D. Yuan, "Analysis of cell load coupling for LTE network planning and optimization", IEEE Transactions on Wireless Communications, 11(6): 2287-2297, 2012.

[4] I. Siomina, A. Furuskär, and G. Fodor. "A mathematical framework for statistical QoS and capacity studies in OFDM networks", IEEE International Symposium on Personal, Indoor and Mobile Radio Communications (PIMRC), 2009.

[5] R. L. G. Cavalcante, S. Stanczak, M. Schubert, A. Eisenblaetter, and U. Tuerke, "Toward energy-efficient 5G wireless communications technologies: tools for decoupling the scaling of networks from the growth of operating power", IEEE Signal Processing Magazine, 31(6): 24-34, 2014.

[6] A. J. Fehske, I. Viering, J. Voigt, C. Sartori, S. Redana, and G P. Fettweis, "A mathematical perspective of self-optimizing wireless networks", IEEE International Conference on Communications (ICC), 2009.

[7] A. J. Fehske, H. Klessig, J. Voigt, and G. P. Fettweis, "Concurrent loadaware adjustment of user association and antenna tilts in self-organizing radio networks", IEEE Transactions on Vehicular Technology, 62(5): 1974-1988, 2013.

[8] A. J. Fehske and G. P. Fettweis, "Aggregation of variables in load models for interference-coupled cellular data networks", IEEE International Conference on Communications (ICC), 2012.

[9] R. L. G. Cavalcante, S. Stańczak, M. Schubert, A. Eisenblaetter, and U. Türke, "Toward energy-efficient $5 \mathrm{G}$ wireless communications technologies: Tools for decoupling the scaling of networks from the growth of operating power", IEEE Signal Processing Magazine, 31(6):24-34, 2014.

[10] I. Siomina and D. Yuan, "Optimization approaches for planning small cell locations in load-coupled heterogeneous LTE networks", IEEE International Symposium on Personal Indoor and Mobile Radio Communications (PIMRC), 2013.

[11] I. Siomina and D. Yuan, "Optimizing small cell range in heterogeneous and load-coupled LTE networks", IEEE Transactions on Vehicular Technology, 64(5): 2169-2174, 2014.

[12] L. You, L. Lei, and D. Yuan, "Load balancing via joint transmission in heterogeneous LTE: Modeling and computation", IEEE International Symposium on Personal Indoor and Mobile Radio Communications (PIMRC), 2015.
[13] G. Athanasiou, P. C. Weeraddana, C. Fischione, and L. Tassiulas, “Optimizing client association for load balancing and fairness in millimeterwave wireless networks", IEEE/ACM Transactions on Networking, 23(3): 836-850, 2015.

[14] R. Sun, M. Hong, and Z. Luo, "Joint downlink base station association and power control for max-min fairness: computation and complexity", IEEE Journal on Selected Areas of Communications, 33(6): 1040-1054, 2015.

[15] A. G. Gotsis, S. Stefanatos, and A. Alexiou, Optimal user association for massive MIMO empowered ultra-dense wireless networks, International Conference on Communication Workshop (ICCW), 2015

[16] S. Singh, F. Baccelli, and J. G. Andrews, "On association cells in random heterogeneous networks", IEEE Wireless Communications Letters, 3(1):70-73, 2014

[17] Q. Li, R. Q. Hu, Y. Qian, and G. Wu, "Intracell cooperation and resource allocation in a heterogeneous network with relays", IEEE Transactions on Vehicular Technology, 62(4): 1770-1784, 2013.

[18] M. Hong and A. Garcia, "Mechanism design for base station association and resource allocation in downlink OFDMA network", IEEE Journal on Selected Areas of Communications, 30(11): 22382250, 2012.

[19] Q. Ye, B. Rong, Y. Chen, M. Al-Shalash, C. Caramanis, and J. G. Andrews, "User association for load balancing in heterogeneous cellular networks". IEEE Transactions on Wireless Communications, 12(6): 2706-2716, 2013.

[20] G. Nigam, P. Minero, and M. Haenggi. "Coordinated multipoint joint transmission in heterogeneous networks", IEEE Transactions on Communications, 62(11): 4134-4146, 2014.

[21] T. R. Lakshmana and B. Makki, "Frequency allocation in non-coherent joint transmission CoMP networks", IEEE International Conference on Communications (ICC), 2014.

[22] R. Tanbourgi, S. Singh, J. G. Andrews, and F. K. Jondral, "A tractable model for noncoherent joint-transmission base station cooperation", IEEE Transactions on Wireless Communications, 13(9): 4959-4973, 2014.

[23] F. Baccelli and A. Giovanidis, "A stochastic geometry framework for analyzing pairwise-cooperative cellular networks", IEEE Transactions on Wireless Communications, 14(2):794-808, 2015.

[24] M. Schubert and H. Boche, A General Framework for Interference Management and Network Utility Optimization, Springer, 2014.

[25] S. Boyd and L. Vandenberghe, Convex Optimization, Cambridge, 2014.

[26] R. D. Yates, "A framework for uplink power control in cellular radio systems". IEEE Journal on Selected Areas in Communications, 13(7): 1341-1347, 1995.

[27] C. Y. Huang and R. D. Yates, "Rate of convergence for minimum power assignment algorithms in cellular radio systems", Wireless Networks, 4(4):223-231, 1998

[28] 3GPP TR 36.913, V13.0.0, 2016

[29] 3GPP TR 36.814, V9.0.0, 2010. 